TRANSACTIONS OF THE

AMERICAN MATHEMATICAL SOCIETY

Volume 363, Number 12, December 2011, Pages 6351-6384

S 0002-9947(2011)05267-0

Article electronically published on July 26, 2011

\title{
A FOURIER TRANSFORM APPROACH TO CHRISTOFFEL'S PROBLEM
}

\author{
PAUL GOODEY, VLADYSLAV YASKIN, AND MARYNA YASKINA
}

\begin{abstract}
We use Fourier transform techniques to provide a new approach to Berg's solution of the Christoffel problem. This leads to an explicit description of Berg's spherical kernel and to new regularity properties of the associated integral transform.
\end{abstract}

\section{INTRODUCTION}

The background for our work comprises the Christoffel-Minkowski problem. To describe this, we recall that associated with a convex body $K \subset \mathbb{R}^{n}$ are its surface area measures $S_{j}(K ; \cdot)$ for $j=1, \ldots, n-1$. Each of these is a positive measure on $S^{n-1}$ which determines the body $K$ up to translation; see [31] for their definitions and properties. For $1 \leq j \leq n-1$, the general Christoffel-Minkowski problem asks for a characterization of those measures on $S^{n-1}$ which are the $j$-th surface area measures of a convex body. The case $j=1$ is known as the Christoffel problem and the case $j=n-1$ is the Minkowski problem.

For convex bodies $K$ having positive curvature and $C^{2}$ boundary, the measure $S_{j}(K ; \cdot)$ has a density (with respect to spherical Lebesgue measure) which is given by the $j$-th normalized elementary symmetric function of the principal radii of curvature:

$$
s_{j}(\xi)=\left(\begin{array}{c}
n-1 \\
j
\end{array}\right)^{-1} \sum_{1 \leq i_{1} \leq \cdots \leq i_{j} \leq n-1} R_{i_{1}}(\xi) \cdots R_{i_{j}}(\xi),
$$

where $R_{1}(\xi), \ldots R_{n-1}(\xi)$ are the principal radii of curvature at the boundary point of $K$ with the outward normal $\xi \in S^{n-1}$.

In particular,

$$
s_{1}(\xi)=\frac{1}{n-1}\left(R_{1}(\xi)+\cdots+R_{n-1}(\xi)\right)
$$

is the mean radius of curvature, and

$$
s_{n-1}(\xi)=R_{1}(\xi) \cdots R_{n-1}(\xi)
$$

is the reciprocal Gauss curvature.

These surface area measures are special cases of the more general mixed surface area measures. It follows, therefore, from [31, equation (5.1.28)] that their centroid is at the origin. Their geometric properties also quickly imply that they cannot be concentrated on any great subsphere of $S^{n-1}$. Consequently any solution of the

Received by the editors October 27, 2009.

2010 Mathematics Subject Classification. Primary 52A20, 42B10, 33C55.

(C)2011 American Mathematical Society Reverts to public domain 28 years from publication 
Christoffel-Minkowski problem must require that the measure have centroid at the origin and not be concentrated on a great subsphere.

In fact, the above conditions suffice for the Minkowski problem. Minkowski's Existence Theorem can be stated as follows: Let $\mu$ be a positive Borel measure on $S^{n-1}$ which is not concentrated on a subsphere. Then, $\mu=S_{n-1}(K ; \cdot)$ for some convex body $K \subset \mathbb{R}^{n}$ if and only if

$$
\int_{S^{n-1}} u \mu(d u)=o
$$

In the case of polytopes, this theorem was obtained by Minkowski; see [26] and 27. His result was subsequently extended to the general setting by Aleksandrov [2] and independently by Fenchel and Jessen [8].

There is extensive literature on the Minkowski Problem. It concerns the above result and its variants. In particular, this includes stability and regularity versions. For further information, we refer the reader to Cheng and Yau [6], Gluck [13] and Pogorelov 28.

In its simplest form, the Christoffel problem asks for necessary and sufficient conditions for a given measure $\mu$ on the sphere $S^{n-1}$ to be the first surface area measure of a convex body $K \subset \mathbb{R}^{n}$. It follows from our observations above that first surface area measures are also $(n-1)$-st surface area measures. However, the conditions of Minkowski's Theorem are no longer sufficient. In particular, $S_{n-1}(P ; \cdot)$, for a polytope $P \subset \mathbb{R}^{n}$ cannot be a first surface area measure.

The problem has a long history. It is named in recognition of Christoffel's work [7, however Alexandrov [1] and [3] pointed to gaps in his work. As Firey [9] points out, these gaps arose since the conditions derived by Christoffel were not sufficient to guarantee that the resultant surface was a closed convex surface. Busemann [5] provides a description of the problem and a number of unsuccessful attempts, though even here there are also some mistakes. The problem, as described above, was eventually solved independently by Firey [9, 10] and Berg [4. The solutions provided by Berg and Firey do not yield a characterization which is easy to apply to specific problems. However, a completely different, and applicable, solution to the polytopal version of the problem was provided by Schneider [30. An approach to Berg's solution in the case of centrally symmetric bodies can be found in [14. Our results will shed new light on Berg's solution to the Christoffel problem and we thereby obtain a new regularity result.

Before embarking on this we note that, in recent times, a great deal of progress has been made on the general Christoffel-Minkowski problem. Much of this is in the context of smooth convex bodies. As we have seen, the problem, then, is to characterize those functions $f$ on $S^{n-1}$ which correspond to the $j$-th elementary symmetric function of the principal radii of curvature of the surface of a convex body. It is, furthermore, important to estimate the smoothness of the body $K$ based on the smoothness properties of $f$. We refer the reader to the papers [18, [19], 20], 21], 22], 32] and the references therein for the recent developments on this problem.

In his paper, Berg constructs certain functions $g_{n}$ on the interval $[-1,1]$ and shows (see [4, Theorem 5.3]) that a given positive Borel measure $\mu$ on the sphere $S^{n-1}$ is the first area measure of a convex body in $\mathbb{R}^{n}$ if and only if the centroid of 
the measure is located at the origin, and the spherical potential defined by

$$
S^{\mu}(\xi)=\int_{S^{n-1}} g_{n}((\theta, \xi)) \mu(d \theta) \quad\left(\xi \in S^{n-1}\right)
$$

is a support function. Here, and subsequently, $(\theta, \xi)$ denotes the usual scalar product of vectors in $\mathbb{R}^{n}$.

Berg gives explicit formulations of the functions $g_{n}$ only for low dimensions $n$, otherwise they are described using recursion relations. Specifically, he shows that, for $t \in(-1,1)$,

$$
\begin{aligned}
& g_{2}(t)=\frac{1}{\pi}\left(\pi-\cos ^{-1} t\right)\left(1-t^{2}\right)^{1 / 2}-\frac{1}{2 \pi} t, \\
& g_{3}(t)=1+t \ln (1-t)+\left(\frac{4}{3}-\ln 2\right) t
\end{aligned}
$$

and, for $n \geq 2$,

$$
g_{n+2}(t)=\frac{n+1}{(n-1)^{2}} t g_{n}^{\prime}(t)+\frac{n+1}{n-1} g_{n}(t)+\frac{1}{\sqrt{\pi}} \frac{(n+1) \Gamma((n+2) / 2)}{(n+2) \Gamma((n+1) / 2)} t .
$$

He also establishes the integrability properties of $g_{n}$ as a function on $[-1,1]$; see [4, Theorem 3.3].

Firey's solution [9] to the smooth case of Christoffel's problem uses the Green's function method to solve a Poisson equation for the position function of the boundary surface of $K$. He then shows that the positivity of a certain integral transform is the characteristic property for a given function to be the mean radius of curvature of a closed convex surface.

In this paper, we will re-create Berg's result using Fourier transform techniques. In order to do this, rather than describing $g_{n}$ as a function on $[-1,1]$, we will fix $\xi \in S^{n-1}$ or $\mathbb{R}^{n}$ and then describe $g_{n}((\cdot, \xi))$ as a function on $S^{n-1}$ or $\mathbb{R}^{n}$. This will result in a much more explicit expression for the functions $g_{n}$.

Theorem. The measure $\mu$ on $S^{n-1}$ is the first surface area measure of a convex body in $\mathbb{R}^{n}$ if and only if it is not supported on a great subsphere, has centroid at the origin, and

$$
\int_{S^{n-1}} g_{n}((\theta, \xi)) \mu(d \theta) \quad\left(\xi \in \mathbb{S}^{n-1}\right)
$$

is a support function, where, for even dimensions $n$, and $\theta \in \mathbb{R}^{n}$,

$$
g_{n}((\theta, \xi))=(2 \pi)^{-n} \frac{2 \pi^{n / 2}}{\Gamma(n / 2)}\left(-\Delta_{\theta}\right)^{\frac{n-2}{2}}\left[|\theta|^{-1}(\pi-\arccos s) \sqrt{1-s^{2}}\right],
$$

whereas, for odd $n$,

$$
g_{n}((\theta, \xi))=(2 \pi)^{-n} \frac{\pi^{\frac{n+1}{2}}}{\Gamma((n+1) / 2)}\left(-\Delta_{\theta}\right)^{\frac{n-1}{2}}[s \ln (1-s)-\ln (1-s)-\ln |\theta|] .
$$

Here, $s$ denotes $(\theta /|\theta|, \xi)$ and, in the above expressions for $g_{n}$, it is assumed that $\theta \notin \operatorname{span}\{\xi\}$.

Our formulation of the $g_{n}$ 's differs slightly from that of Berg. We have taken advantage of the fact that the measure $\mu$ has centroid at the origin to ignore multiples of $s$ in our expression for $g_{n}(s)$. 
The techniques we use are based on those introduced by Koldobsky to solve a variety of geometric problems; see 24]. These show how certain spherical integral transforms can be obtained by applying Fourier transforms to homogeneous extensions of the spherical functions. For the vast majority of these applications, the transforms are restricted to even spherical functions. In [15], we extended these transform results to general spherical functions and this extension will play a key role in the current paper. These techniques will also allow us to establish new regularity results which, in turn, have implications for certain stability results.

In $\S 2$, we will collect the results and background information on which our techniques are based. The main ideas will be presented in $\S 3$ in the context of smooth bodies. The detailed calculation of the Fourier transforms of various distributions will be carried out in $\S 4$ and the proof of the main result will be the content of $\S 5$. The final section of the paper will provide a regularity result explaining how smoothness properties of the measure $\mu$ carry over to smoothness of the body with first surface area measure $\mu$. We are grateful to Markus Kiderlen for his comments on an earlier version of this manuscript.

\section{Definitions AND PRELIMINARY RESUlts}

We denote by $\mathcal{S}$ the Schwartz space of rapidly decreasing infinitely differentiable functions on $\mathbb{R}^{n}$. Elements of this space will be called test functions. For $\phi \in \mathcal{S}$, the Fourier transform of $\phi$ is defined by

$$
\hat{\phi}(x)=\int_{\mathbb{R}^{n}} \phi(y) e^{-i(x, y)} d y \quad \text { for } x \in \mathbb{R}^{n} .
$$

Distributions are the elements of the dual space, $\mathcal{S}^{\prime}$, of linear continuous functionals on $\mathcal{S}$. We will primarily be dealing with homogeneous distributions on $\mathbb{R}^{n}$. For background information, the reader is referred to the book [11] by Gelfand and Shilov.

For $f \in C^{\infty}\left(S^{n-1}\right)$ and $p \in \mathbb{C}$, we denote by $f_{p}$ the homogeneous degree $-n+p$ extension of $f$ to $\mathbb{R}^{n} \backslash o$. Thus

$$
f_{p}(x)=|x|^{-n+p} f\left(\frac{x}{|x|}\right) \quad \text { for } x \neq o .
$$

Thought of as a distribution, $f_{p}$ has Fourier transform, $\hat{f}_{p}$, which is defined by

$$
\left\langle\hat{f}_{p}, \phi\right\rangle=\left\langle f_{p}, \hat{\phi}\right\rangle
$$

for any test function $\phi$. For example, if $0<\Re p<1, \hat{f}_{p}$ is a function and, for $x \in \mathbb{R}^{n}$,

$$
\begin{aligned}
\hat{f}_{p}(x)=\Gamma(p) \cos \frac{p \pi}{2} \int_{S^{n-1}}|(x, \theta)|^{-p} f(\theta) d \theta & \\
& -i \Gamma(p) \sin \frac{p \pi}{2} \int_{S^{n-1}}|(x, \theta)|^{-p} \operatorname{sgn}(x, \theta) f(\theta) d \theta .
\end{aligned}
$$

Consequently, this distribution acts by integration and its action can be extended to include functions $f \in C\left(S^{n-1}\right)$. For $f \in C\left(S^{n-1}\right)$ and $\xi \in S^{n-1}$, we define

$$
F_{\xi}(t)=\left(1-t^{2}\right)^{(n-3) / 2} \int_{S^{n-1} \cap \xi^{\perp}} f\left(t \xi+\sqrt{1-t^{2}} \zeta\right) d \zeta .
$$


In the case $f \in C^{\infty}\left(S^{n-1}\right), F_{\xi}$ is smooth in $(-1,1)$ and equation (11) becomes

$$
\hat{f}_{p}(\xi)=\frac{1}{2}\left\langle\left(|r|^{p-1}\right)^{\wedge}+\left(|r|^{p-1} \operatorname{sgn} r\right)^{\wedge}, F_{\xi}\right\rangle,
$$

for $\xi \in S^{n-1}$; see [15], for example. For non-integer values of $p \in \mathbb{C}$, the distributions $\left(|r|^{p-1}\right)^{\wedge}$ and $\left(|r|^{p-1} \operatorname{sgn} r\right)^{\wedge}$ can be extended to an analytic family of distributions; see [11. In fact, regularization techniques can be used to extend them to many integer values of $p$. This was used in [15] to show, for example, that for $\xi \in S^{n-1}$,

$$
\begin{aligned}
\hat{f}_{-1}(\xi)= & -\frac{\pi}{2} \int_{S^{n-1}}|(u, \xi)| f(u) d u \\
& -i \int_{S^{n-1}}\left(1+\Gamma^{\prime}(1)-\ln |(u, \xi)|\right)(u, \xi) f(u) d u \\
\hat{f}_{0}(\xi)=\int_{S^{n-1}}\left(\Gamma^{\prime}(1)-\ln |(u, \xi)|\right) f(u) d u & \quad+i \pi\left(\frac{1}{2} \int_{S^{n-1}} f(u) d u-\int_{S^{n-1} \cap \xi^{+}} f(u) d u\right), \\
\hat{f}_{1}(\xi)=\lim _{p \rightarrow 1} \hat{f}_{p}(\xi) \quad f(u) d u-i \int_{-1}^{1} t^{-1}\left(F_{\xi}(t)-F_{\xi}(0)\right) d t . & \pi \int_{S^{n-1} \cap \xi^{\perp}}
\end{aligned}
$$

Again, a number of these distributions can be extended to larger classes of functions $f$. In particular, the equations (2), (3) and, for even functions, (5) hold for $f \in$ $C\left(S^{n-1}\right)$.

The first step in our formulation of the functions $g_{n}$ will reduce the problem to calculating the Fourier transforms of the functions $|(x, \xi)||x|^{-2}$ and $(x, \xi)|x|^{-2} \ln |(x /|x|, \xi)|$ as functions of $x \in \mathbb{R}^{n}$. These are functions of homogeneity -1 and thus do not fit immediately into the results described above. However, the well-known relationship between Fourier transforms and differentiation quickly addresses this problem. We have

$$
(\Delta f)^{\wedge}(x)=-|x|^{2} \hat{f}(x) \quad \text { and } \quad \Delta \hat{f}(x)=-\left(|\cdot|^{2} f\right)^{\wedge}(x) ;
$$

see Rudin [29, Theorem 7.4] or Koldobsky [24, page 35], for example. It follows for even $n$, for example, that

$$
\left(|(x, \xi)||x|^{-2}\right)^{\wedge}=(-1)^{(n-2) / 2} \Delta^{(n-2) / 2}\left(|(x, \xi)||x|^{-n}\right)^{\wedge} .
$$

Thus, for even dimensions $n$, it will suffice to calculate the Fourier transform of $|(x, \xi)||x|^{-n}$. This is the homogeneous degree $-n+1$ extension of a continuous function on $S^{n-1}$ and thus (5) can be used to calculate the Fourier transform. Similar ideas will allow us to use (2), (3) and (5) to find $g_{n}$ in all dimensions $n$.

As mentioned in the introduction, we also wish to establish some regularity results. For these, we will make use of the equations (3.8) and (3.9) from [15]. If $p$ is a non-positive even integer and $f \in C^{\infty}\left(S^{n-1}\right)$ is even,

$$
\hat{f}_{p}(\xi)=(-1)^{p / 2} \frac{\pi}{(-p) !} \int_{S^{n-1}}\left(C_{p}-\ln |(u, \xi)|\right)|(u, \xi)|^{-p} f(u) d u
$$


for a constant $C_{p}$ dependent only on $p$, whereas, if $p$ is a negative odd integer and $f$ is even,

$$
\hat{f}_{p}(\xi)=(-1)^{(p-1) / 2} \frac{\pi}{2(-p) !} \int_{S^{n-1}}|(u, \xi)|^{-p} f(u) d u .
$$

We note that both (8) and (9) are true for functions $f \in C\left(S^{n-1}\right)$ and that they hold for $\xi \in \mathbb{R}^{n}$.

\section{Smooth CONVEx Bodies}

As already explained, the Christoffel problem asks for necessary and sufficient conditions on a measure $\mu$ on $S^{n-1}$ for it to be the first surface area measure, $S_{1}(K ; \cdot)$, of a convex body in $\mathbb{R}^{n}$.

Initially, we will assume that $K$ is of class $C_{+}^{2}$; for this and other notions in convex geometry, we refer to [31. It follows that the support function, $h$, of $K$ is $C^{2}$. For $\xi \in S^{n-1}$, we put

$$
f(\xi)=R_{1}(\xi)+\cdots+R_{n-1}(\xi)
$$

where the $R_{i}(\xi)$ are the principal radii of curvature of $K$ at the point on the boundary of $K$ with the outward normal $\xi$. Thus $f$ is (up to a normalization factor) the first elementary symmetric function of the radii of curvature. We next extend $f$ from the sphere to a homogeneous, degree -1 function on $\mathbb{R}^{n} \backslash o$. Christoffel's problem then reduces to solving the equation (see, for example, [31, p. 109])

$$
\Delta h(x)=f(x), \quad x \in \mathbb{R}^{n} .
$$

Using Green's Formula, we easily deduce the following necessary condition for $f$ to be the first elementary symmetric function of the radii of curvature of a convex body:

$$
\int_{S^{n-1}} x f(x) d x=o, \quad \text { equivalently } \int_{S^{n-1}}(x, \xi) f(x) d x=0,
$$

for all $\xi \in S^{n-1}$. As mentioned in the introduction, this condition also suffices to guarantee that $f$ is the $(n-1)$-st surface area measure of a convex body in $\mathbb{R}^{n}$. However, the characterization of first surface area measures requires further conditions.

Applying the Fourier transform (in the sense of distributions) to equation (10), we get

$$
-|x|^{2} \widehat{h}(x)=\widehat{f}(x)
$$

see (6). Now let $\phi$ be a test function whose Fourier transform is supported outside of the origin. Clearly, $|x|^{-2} \hat{\phi}(x)$ is also a test function and

$$
-\left\langle|x|^{2} \widehat{h}(x),|x|^{-2} \hat{\phi}(x)\right\rangle=\left\langle\widehat{f}(x),|x|^{-2} \hat{\phi}(x)\right\rangle .
$$

Therefore,

$$
-\langle\widehat{h}(x), \hat{\phi}(x)\rangle=\left\langle|x|^{-2} \widehat{f}(x), \hat{\phi}(x)\right\rangle,
$$

and so

$$
-\langle\hat{\hat{h}}, \phi\rangle=\left\langle\left(|x|^{-2} \hat{f}(x)\right)^{\wedge}, \phi\right\rangle .
$$

This equality is true for all test functions whose Fourier transform is supported outside of the origin, and so $\hat{\hat{h}}$ and $-\left(|x|^{-2} \hat{f}(x)\right)^{\wedge}$ differ only by a polynomial; see 
[29. 7.16]. In view of the homogeneity, this polynomial is a linear function and therefore,

$$
\hat{\hat{h}}(\xi)=-\left(|x|^{-2} \hat{f}(x)\right)^{\wedge}(\xi)+(\xi, a)
$$

for some $a \in \mathbb{R}^{n}$. It is not hard to see that $a$ is (a multiple of) the Steiner point of the body $K$. However, knowledge of the radii of curvature gives no information about the position of the body and so we can assume that the Steiner point of the body is located at the origin. This allows us to ignore the linear term.

Let $h^{e}, h^{o}, f^{e}, f^{o}$ denote the even and odd parts of the functions $h$ and $f$. Then $\hat{\hat{h}^{e}}=(2 \pi)^{n} h^{e}$ and $\hat{h^{o}}=-(2 \pi)^{n} h^{o}$. Therefore,

$$
(2 \pi)^{n} h^{e}(\xi)=-\left(|x|^{-2} \hat{f}^{e}(x)\right)^{\wedge}(\xi)
$$

and

$$
(2 \pi)^{n} h^{o}(\xi)=\left(|x|^{-2} \hat{f}^{o}(x)\right)^{\wedge}(\xi) .
$$

For the moment we assume that $f$ is an infinitely differentiable function on $\mathbb{R}^{n} \backslash o$. Then $\hat{f}$ is also infinitely differentiable on $\mathbb{R}^{n} \backslash o$ (see, for example, Corollary 3.3 of [15]) and it has homogeneity $-n+1$. Thus $|x|^{-2} \hat{f}^{e}(x)$ is even and homogeneous of degree $-n-1$, therefore, using the real part of (2), we obtain

$$
(2 \pi)^{n} h^{e}(\xi)=\frac{\pi}{2} \int_{S^{n-1}}|(x, \xi)||x|^{-2} \hat{f}^{e}(x) d x .
$$

On the other hand, the function $|x|^{-2} \hat{f}^{o}(x)$ is odd and homogeneous of degree $-n-1$, therefore, the imaginary part of (2) gives

$$
(2 \pi)^{n} h^{o}(\xi)=-i \int_{S^{n-1}}\left(1+\Gamma^{\prime}(1)-\ln |(x, \xi)|\right)(x, \xi)|x|^{-2} \hat{f}^{o}(x) d x .
$$

Using (11) and the fact that $(x, \xi) f^{e}(x)$ is an odd function, we deduce that

$$
\int_{S^{n-1}}(x, \xi) f^{o}(x) d x=0 \quad \text { for all } \xi \in S^{n-1} .
$$

This means that $f^{o}$ has no harmonics of order 1 in its spherical harmonic expansion. Therefore, the same is true of its Fourier transform, and so

$$
\int_{S^{n-1}}(x, \xi) \hat{f}^{o}(x) d x=0 \quad \text { for all } \xi \in S^{n-1} .
$$

Therefore,

$$
\begin{aligned}
(2 \pi)^{n} h^{o}(\xi) & =i \int_{S^{n-1}}\left((x, \xi)|x|^{-2} \ln |(x, \xi)|\right) \hat{f}^{o}(x) d x \\
& =i \int_{S^{n-1}}\left((x, \xi)|x|^{-2} \ln |(x /|x|, \xi)|\right) \hat{f}^{o}(x) d x
\end{aligned}
$$

In order to proceed, we would like to remove the Fourier transform from $f$ in (14) and (15). This requires an analogue of Parseval's formula. However, before this can be achieved, it is necessary to study the Fourier transforms

$$
\left(|(x, \xi)||x|^{-2}\right)^{\wedge} \quad \text { and } \quad\left((x, \xi)|x|^{-2} \ln |(x /|x|, \xi)|\right)^{\wedge} .
$$

In particular, we will show that, for fixed $\xi$, their restrictions to $S^{n-1}$ are integrable there. 


\section{Calculation of Fourier transforms}

The main purpose of this section is to obtain the Fourier transforms in (16). They are given in Theorem 4.1. In $\S 5$, we will combine these results with (14) and (15) to provide a solution to the Christoffel problem.

Theorem 4.1. Let $\xi \in S^{n-1}$ and put $s=(\theta /|\theta|, \xi)$.

1) If $n$ is even,

$$
\begin{gathered}
\left(|(x, \xi)||x|^{-2}\right)^{\wedge}(\theta)=\frac{2 \pi^{n / 2}}{\Gamma(n / 2)}(-1)^{\frac{n-2}{2}} \Delta_{\theta}^{\frac{n-2}{2}}\left(|\theta|^{-1} \sqrt{1-s^{2}}\right) \\
\text { and } \\
\left((x, \xi)|x|^{-2} \ln \left|\left(\frac{x}{|x|}, \xi\right)\right|\right)^{\wedge}(\theta) \\
=i(-1)^{\frac{n}{2}} \Delta_{\theta}^{\frac{n-2}{2}}\left[|\theta|^{-1}\left(C s+\frac{2 \pi^{n / 2}}{\Gamma(n / 2)}\left(\frac{\pi}{2}-\arccos s\right) \sqrt{1-s^{2}}\right)\right]
\end{gathered}
$$

for some constant $C$.

2) If $n$ is odd,

$$
\begin{aligned}
& \left(|(x, \xi)||x|^{-2}\right)^{\wedge}(\theta)=\frac{(-1)^{\frac{n+1}{2}} \pi^{\frac{n-1}{2}}}{\Gamma((n+1) / 2)} \Delta_{\theta}^{\frac{n-1}{2}}\left[\ln \left(1-s^{2}\right)+s \ln \frac{1+s}{1-s}+2 \ln |\theta|\right] \\
& \text { and } \\
& \left((x, \xi)|x|^{-2} \ln \left|\left(\frac{x}{|x|}, \xi\right)\right|\right)^{\wedge}(\theta) \\
& =i(-1)^{\frac{n+1}{2}} \Delta_{\theta}^{\frac{n-1}{2}}\left(C s+\frac{\pi^{\frac{n+1}{2}}}{2 \Gamma\left(\frac{n+1}{2}\right)}\left[s \ln \left(1-s^{2}\right)+\ln \frac{1+s}{1-s}\right]\right)
\end{aligned}
$$

for some constant $C$.

Proof. It is easy to check that $\Delta_{\theta} s$ is a multiple of $s$; see equation (18) below, for example. Thus, since we will be applying these Fourier transforms in the context of (14) and (15), linear terms in $s$ will be annihilated. It is therefore unnecessary to calculate the constants $C$ mentioned in the statement of the theorem.

We will have four different cases, according to whether $n$ is even or odd and to which Fourier transform we are considering.

Case 1. $\left(|(x, \xi)||x|^{-2}\right)^{\wedge}$ and $n$ even.

Instead of computing the Fourier transform of $|(x, \xi)||x|^{-2}$, it is more convenient, first, to compute the Fourier transform of $|(x, \xi)||x|^{-n}$. For this, we apply (5) to the even continuous function $f=|(\xi, \cdot)|$. Thus

$$
\left(|(x, \xi)||x|^{-n}\right)^{\wedge}(\theta)=\pi \int_{S^{n-1} \cap \theta^{\perp}}|(u, \xi)| d u, \quad \theta \in S^{n-1} .
$$

The integrand is the length of the projection of a unit line segment in direction $\xi$ onto a line in the direction $u$. The integral can therefore be calculated using Cauchy's surface area formula; see [31, page 295], for example. Thus

$$
\left(|(x, \xi)||x|^{-n}\right)^{\wedge}(\theta)=\frac{2 \pi^{n / 2}}{\Gamma(n / 2)} \sqrt{1-(\theta, \xi)^{2}}, \quad \theta \in S^{n-1} .
$$


In order to compute the Fourier transform of $|(x, \xi)||x|^{-2}$, we use the connection between the Fourier transform and differentiation; see (6) and (7). First extend the above function to $\mathbb{R}^{n}$ as a homogeneous function of degree -1 and then apply $-\Delta$ (in the distributional sense) the appropriate number of times:

$$
\left(|(x, \xi)||x|^{-2}\right)^{\wedge}(\theta)=\frac{2 \pi^{n / 2}}{\Gamma(n / 2)}(-1)^{\frac{n-2}{2}} \Delta_{\theta}^{\frac{n-2}{2}}\left(|\theta|^{-1} \sqrt{1-s^{2}}\right) .
$$

Case 2. $\left((x, \xi)|x|^{-2} \ln |(x /|x|, \xi)|\right)^{\wedge}$ and $n$ even.

Here, we first compute the Fourier transform

$$
\left((x, \xi)|x|^{-n} \ln \left|\left(\frac{x}{|x|}, \xi\right)\right|\right)^{\wedge}(\theta) .
$$

The function $f(x)=(x, \xi)|x|^{-n} \ln |(x /|x|, \xi)|$ is continuous at all points $x \neq o$. We will use (11) and (4) to evaluate $\hat{f}_{1}$. Thus

$$
\begin{aligned}
\left((x, \xi)|x|^{-n} \ln \left|\left(\frac{x}{|x|}, \xi\right)\right|\right)^{\wedge}(\theta)=\lim _{\epsilon \rightarrow 0^{+}}\left((x, \xi)|x|^{-n-\epsilon} \ln \left|\left(\frac{x}{|x|}, \xi\right)\right|\right)^{\wedge}(\theta) & \\
= & -i \lim _{\epsilon \rightarrow 0^{+}} \int_{S^{n-1}}|(u, \theta)|^{-1+\epsilon} \operatorname{sgn}(u, \theta)(u, \xi) \ln |(u, \xi)| d u=:-i I_{n} .
\end{aligned}
$$

Now let us make the following change of variables:

$$
u=t w+\sqrt{1-t^{2}} v
$$

where $w$ is any unit vector, orthogonal to both $\xi$ and $\theta, v \in S^{n-1} \cap w^{\perp}$, and $t \in[-1,1]$. Then, for some constant $C$ dependent only on the dimension $n$,

$$
\begin{aligned}
I_{n}= & \lim _{\epsilon \rightarrow 0^{+}} \int_{-1}^{1}\left(1-t^{2}\right)^{\frac{n-3+\epsilon}{2}} \int_{S^{n-1} \cap w^{\perp}}|(v, \theta)|^{-1+\epsilon} \operatorname{sgn}(v, \theta) \\
& \times(v, \xi) \ln \left|\sqrt{1-t^{2}}(v, \xi)\right| d v d t \\
= & C \lim _{\epsilon \rightarrow 0^{+}} \int_{S^{n-1} \cap w^{\perp}}|(v, \theta)|^{-1+\epsilon} \operatorname{sgn}(v, \theta)(v, \xi) d v \\
& +\int_{-1}^{1}\left(1-t^{2}\right)^{\frac{n-3}{2}} d t \lim _{\epsilon \rightarrow 0^{+}} \int_{S^{n-1} \cap w^{\perp}}|(v, \theta)|^{-1+\epsilon} \operatorname{sgn}(v, \theta)(v, \xi) \ln |(v, \xi)| d v .
\end{aligned}
$$

The first integral in the latter expression is just the Fourier transform of $(v, \xi)|v|^{-n+1}$ in the subspace $w^{\perp}$, which equals $C \cdot(\theta, \xi)$, since spherical harmonics are mapped to spherical harmonics; see [15].

Therefore we obtain a recurrence relation,

$$
I_{n}=C \cdot(\theta, \xi)+\frac{\sqrt{\pi} \Gamma((n-1) / 2)}{\Gamma(n / 2)} I_{n-1},
$$

where $I_{n-1}$ is the analogous integral in the lower-dimensional setting. Thus

$$
I_{n}=C \cdot(\theta, \xi)+\frac{\pi^{(n-2) / 2}}{\Gamma(n / 2)} I_{2}
$$

here, and in the sequel, $C$ denotes a (variable) constant dependent only on $n$.

The question is therefore reduced to computing

$$
I_{2}=\lim _{\epsilon \rightarrow 0^{+}} \int_{S^{1}}|(v, \theta)|^{-1+\epsilon} \operatorname{sgn}(v, \theta)(v, \xi) \ln |(v, \xi)| d v .
$$


Let $\alpha$ be the angle between $\xi$ and $\theta$. Introducing a new variable $\phi$, we get

$$
\begin{aligned}
I_{2} & =\lim _{\epsilon \rightarrow 0^{+}} \int_{0}^{2 \pi}|\cos \phi|^{-1+\epsilon} \operatorname{sgn}(\cos \phi) \ln |\cos (\phi-\alpha)| \cos (\phi-\alpha) d \phi \\
& =\lim _{\epsilon \rightarrow 0^{+}} \cos \alpha \int_{0}^{2 \pi}|\cos \phi|^{-1+\epsilon} \operatorname{sgn}(\cos \phi) \ln |\cos (\phi-\alpha)| \cos \phi d \phi \\
& +\lim _{\epsilon \rightarrow 0^{+}} \sin \alpha \int_{0}^{2 \pi}|\cos \phi|^{-1+\epsilon} \operatorname{sgn}(\cos \phi) \ln |\cos (\phi-\alpha)| \sin \phi d \phi .
\end{aligned}
$$

Let us call the latter two expressions $J_{1}$ and $J_{2}$, respectively. Then

$$
\begin{gathered}
J_{1}=\lim _{\epsilon \rightarrow 0^{+}} \cos \alpha \int_{0}^{2 \pi}|\cos \phi|^{-1+\epsilon} \operatorname{sgn}(\cos \phi) \ln |\cos (\phi-\alpha)| \cos \phi d \phi \\
=\cos \alpha \int_{0}^{2 \pi} \ln |\cos (\phi-\alpha)| d \phi=C \cos \alpha,
\end{gathered}
$$

since the integral does not depend on $\alpha$.

To compute $J_{2}$, we add the same integral with $\phi$ replaced by $-\phi$ :

$$
J_{2}=\frac{\sin \alpha}{2} \lim _{\epsilon \rightarrow 0^{+}} \int_{0}^{2 \pi}|\cos \phi|^{-1+\epsilon} \operatorname{sgn}(\cos \phi) \ln \left|\frac{\cos (\phi-\alpha)}{\cos (\phi+\alpha)}\right| \sin \phi d \phi .
$$

Now there is no problem with the convergence of this integral for $\epsilon=0$ because of the zero in the logarithmic term. So,

$$
\begin{gathered}
J_{2}=\frac{\sin \alpha}{2} \int_{0}^{2 \pi} \frac{\sin \phi}{\cos \phi} \ln \left|\frac{\cos (\phi-\alpha)}{\cos (\phi+\alpha)}\right| d \phi \\
=\sin \alpha \int_{-\pi / 2}^{\pi / 2} \frac{\sin \phi}{\cos \phi} \ln \left|\frac{\cos \phi \cos \alpha+\sin \phi \sin \alpha}{\cos \phi \cos \alpha-\sin \phi \sin \alpha}\right| d \phi .
\end{gathered}
$$

After the substitution $z=\tan \phi$, we get

$$
J_{2}=\sin \alpha \int_{-\infty}^{\infty} \frac{z}{1+z^{2}} \ln \left|\frac{\cos \alpha+z \sin \alpha}{\cos \alpha-z \sin \alpha}\right| d z
$$

One can compute this integral by complex contour integration. Instead, we will use integration by parts to write this integral in a different form:

$$
J_{2}=-\sin ^{2} \alpha \cos \alpha \int_{-\infty}^{\infty} \frac{\ln \left(1+z^{2}\right)+\ln \sin ^{2} \alpha}{\cos ^{2} \alpha-z^{2} \sin ^{2} \alpha} d z
$$

and then use formula 4.295 (8) from Gradshteyn and Ryzhik, [16, p. 560] to get

$$
J_{2}=2 \pi\left(\frac{\pi}{2}-\alpha\right) \sin \alpha .
$$

Therefore,

$$
I_{n}=C \cdot(\theta, \xi)+\frac{\pi^{(n-2) / 2}}{\Gamma(n / 2)} 2 \pi\left(\frac{\pi}{2}-\alpha\right) \sin \alpha .
$$

So,

$$
I_{n}=C \cdot s+\frac{2 \pi^{n / 2}}{\Gamma(n / 2)}\left(\frac{\pi}{2}-\arccos s\right) \sqrt{1-s^{2}} .
$$


As previously mentioned, in order to obtain the Fourier transform that we need, we have to extend the latter to $\mathbb{R}^{n}$ as a homogeneous function of degree -1 and then apply the Laplacian.

$$
\begin{aligned}
\left((x, \xi)|x|^{-2} \ln \left|\left(\frac{x}{|x|}, \xi\right)\right|\right)^{\wedge}(\theta) \\
\quad=i(-1)^{\frac{n}{2}} \Delta_{\theta}^{\frac{n-2}{2}}\left[|\theta|^{-1}\left(C s+\frac{2 \pi^{n / 2}}{\Gamma(n / 2)}\left(\frac{\pi}{2}-\arccos s\right) \sqrt{1-s^{2}}\right)\right] .
\end{aligned}
$$

Case 3. $\left(|(x, \xi)||x|^{-2}\right)^{\wedge}$ and $n$ odd.

For odd $n$, instead of computing the Fourier transform of $|(x, \xi)||x|^{-2}$ we will compute the Fourier transform of $|(x, \xi)||x|^{-n-1}$. Here, we apply formula (3) to the continuous function $f=|(\xi, \cdot)|$. This gives

$$
\left(|(x, \xi)||x|^{-n-1}\right)^{\wedge}(\theta)=\int_{S^{n-1}}\left(\Gamma^{\prime}(1)-\ln |(u, \theta)|\right)|(u, \xi)| d u, \quad \theta \in S^{n-1} .
$$

From the latter expression we need only compute the integral

$$
I_{n}=\int_{S^{n-1}}|(u, \xi)| \ln |(u, \theta)| d u,
$$

since the other term is a constant, which will subsequently be annihilated by the Laplacian.

To this end, make the following change of variables:

$$
u=t w+\sqrt{1-t^{2}} v,
$$

where $w$ is any unit vector, orthogonal to both $\xi$ and $\theta, v \in S^{n-1} \cap w^{\perp}$, and $t \in[-1,1]$. Then

$$
\begin{gathered}
I_{n}=\int_{-1}^{1}\left(1-t^{2}\right)^{\frac{n-3}{2}} \int_{S^{n-1} \cap w^{\perp}} \sqrt{1-t^{2}}|(v, \xi)| \ln \left|\sqrt{1-t^{2}}(v, \theta)\right| d v d t \\
=\int_{-1}^{1}\left(1-t^{2}\right)^{\frac{n-2}{2}} \ln \sqrt{1-t^{2}} d t \int_{S^{n-1} \cap w^{\perp}}|(v, \xi)| d v \\
+\int_{-1}^{1}\left(1-t^{2}\right)^{\frac{n-2}{2}} d t \int_{S^{n-1} \cap w^{\perp}}|(v, \xi)| \ln |(v, \theta)| d v \\
=C+\frac{\sqrt{\pi} \Gamma(n / 2)}{\Gamma((n+1) / 2)} I_{n-1} .
\end{gathered}
$$

So, using this recurrence formula, we get

$$
I_{n}=C+\frac{\pi^{(n-1) / 2}}{2 \Gamma((n+1) / 2)} I_{2} .
$$

Now we compute the integral $I_{2}$. The integration will take place in the 2dimensional plane spanned by $\xi$ and $\theta$ :

$$
I_{2}=\int_{S^{1}} \ln |(v, \theta)||(v, \xi)| d v .
$$


Making a change of variable we get

$$
I_{2}=\int_{0}^{2 \pi} \ln |\cos \phi||\cos (\phi-\alpha)| d \phi
$$

where $\alpha$ is the angle between $\xi$ and $\theta$.

This integral can be computed by elementary methods (removing the absolute value and integrating by parts). The result is

$$
I_{2}=2 \ln \left(1-s^{2}\right)+2 s \ln \frac{1+s}{1-s}-4,
$$

where $s=\cos \alpha=(\xi, \theta)$.

Therefore,

$$
I_{n}=C+\frac{\pi^{\frac{n-1}{2}}}{\Gamma((n+1) / 2)}\left[\ln \left(1-s^{2}\right)+s \ln \frac{1+s}{1-s}\right]
$$

If $\theta \in \mathbb{R}^{n} \backslash o$, not necessarily a unit vector, then

$$
\begin{gathered}
\left(|(x, \xi)||x|^{-n-1}\right)^{\wedge}(\theta)=C-\int_{S^{n-1}} \ln |(u, \theta)||(u, \xi)| d u \\
=C-\int_{S^{n-1}} \ln \left|\left(u, \frac{\theta}{|\theta|}\right)\right||(u, \xi)| d u-\ln |\theta| \int_{S^{n-1}}|(u, \xi)| d u \\
=C-I_{n}-\frac{2 \pi^{(n-1) / 2}}{\Gamma((n+1) / 2)} \ln |\theta|,
\end{gathered}
$$

as before.

In order to compute the required Fourier transform, we apply the Laplacian

$$
\left(|(x, \xi)||x|^{-2}\right)^{\wedge}(\theta)=\frac{(-1)^{\frac{n+1}{2}} \pi^{\frac{n-1}{2}}}{\Gamma((n+1) / 2)} \Delta_{\theta}^{\frac{n-1}{2}}\left[\ln \left(1-s^{2}\right)+s \ln \frac{1+s}{1-s}+2 \ln |\theta|\right] .
$$

Case $4 .\left((x, \xi)|x|^{-2} \ln |(x /|x|, \xi)|\right)^{\wedge}$ and $n$ odd.

As in the previous cases, it is more convenient to compute

$$
\left((x, \xi)|x|^{-n-1} \ln \left|\left(\frac{x}{|x|}, \xi\right)\right|\right)^{\wedge}
$$

than the Fourier transform of $(x, \xi)|x|^{-2} \ln |(x /|x|, \xi)|$. For this, we apply (3) to the odd continuous function $f=(\xi, \cdot) \ln |(\xi, \cdot)|$. This gives

$$
\left((x, \xi)|x|^{-n-1} \ln \left|\left(\frac{x}{|x|}, \xi\right)\right|\right)^{\wedge}(\theta)=-\frac{i \pi}{2} \int_{S^{n-1}} \operatorname{sgn}(u, \theta)(u, \xi) \ln |(u, \xi)| d u .
$$

Denote the latter integral by $I_{n}$. In order to obtain a recurrence formula, we again make the change of variables:

$$
u=t w+\sqrt{1-t^{2}} v
$$


where $w$ is any unit vector, orthogonal to both $\xi$ and $\theta, v \in S^{n-1} \cap w^{\perp}$, and $t \in[-1,1]$. Then

$$
\begin{aligned}
I_{n}= & \int_{-1}^{1}\left(1-t^{2}\right)^{\frac{n-3}{2}} \int_{S^{n-1} \cap w^{\perp}} \operatorname{sgn}(v, \theta)(v, \xi) \ln \left|\sqrt{1-t^{2}}(v, \xi)\right| d v d t \\
= & C \int_{S^{n-1} \cap w^{\perp}} \operatorname{sgn}(v, \theta)(v, \xi) d v \\
& \quad+\int_{-1}^{1}\left(1-t^{2}\right)^{\frac{n-2}{2}} d t \int_{S^{n-1} \cap w^{\perp}} \operatorname{sgn}(v, \theta)(v, \xi) \ln |(v, \xi)| d v \\
= & C(\xi, \theta)+\frac{\sqrt{\pi} \Gamma(n / 2)}{\Gamma((n+1) / 2)} I_{n-1} .
\end{aligned}
$$

This recurrence formula gives

$$
I_{n}=C(\xi, \theta)+\frac{\pi^{(n-1) / 2}}{2 \Gamma((n+1) / 2)} I_{2} .
$$

Now, if $\alpha$ denotes the angle between $\theta$ and $\xi$,

$$
\begin{aligned}
I_{2}= & \int_{S^{1}} \operatorname{sgn}(v, \theta)(v, \xi) \ln |(v, \xi)| d v \\
= & \int_{0}^{2 \pi} \operatorname{sgn}(\cos (\phi-\alpha)) \cos \phi \ln |\cos \phi| d \phi=2 \int_{-\pi / 2+\alpha}^{\pi / 2+\alpha} \cos \phi \ln |\cos \phi| d \phi \\
& =4 \ln |\sin \alpha| \cos \alpha-4 \cos \alpha+2 \ln \frac{1+\cos \alpha}{1-\cos \alpha},
\end{aligned}
$$

by elementary computations.

Letting $s=\cos \alpha=(\xi, \theta)$, we get

$$
I_{n}=C s+\frac{\pi^{\frac{n-1}{2}}}{\Gamma((n+1) / 2)}\left[s \ln \left(1-s^{2}\right)+\ln \frac{1+s}{1-s}\right] .
$$

Therefore,

$$
\begin{aligned}
\left((x, \xi)|x|^{-n-1} \ln \left|\left(\frac{x}{|x|}, \xi\right)\right|\right)^{\wedge}(\theta) & \\
& =-\frac{i \pi}{2}\left(C s+\frac{\pi^{\frac{n-1}{2}}}{\Gamma((n+1) / 2)}\left[s \ln \left(1-s^{2}\right)+\ln \frac{1+s}{1-s}\right]\right) .
\end{aligned}
$$

Thus

$$
\begin{aligned}
& \left((x, \xi)|x|^{-2} \ln \left|\left(\frac{x}{|x|}, \xi\right)\right|\right)^{\wedge}(\theta) \\
& \quad=i(-1)^{\frac{n+1}{2}} \Delta_{\theta}^{\frac{n-1}{2}}\left(C s+\frac{\pi^{\frac{n+1}{2}}}{2 \Gamma((n+1) / 2)}\left[s \ln \left(1-s^{2}\right)+\ln \frac{1+s}{1-s}\right]\right) .
\end{aligned}
$$

This completes the proof of the theorem. 


\section{The Christoffel Problem}

We will now use (14) and (15) together with Theorem 4.1 to solve Christoffel's problem for smooth convex bodies. We see, from (14) and (15), that

$$
h(\xi)=(2 \pi)^{-n} \int_{S^{n-1}}\left[\frac{\pi}{2}|(x, \xi)||x|^{-2}+i(x, \xi) \ln \left|\left(\frac{x}{|x|}, \xi\right)\right||x|^{-2}\right] \hat{f}(x) d x .
$$

Theorem 4.1 shows that, ignoring the linear terms, we have the following distributional equalities:

$$
\begin{aligned}
{\left[\frac{\pi}{2}|(x, \xi)||x|^{-2}+\right.} & \left.i(x, \xi) \ln \left|\left(\frac{x}{|x|}, \xi\right)\right||x|^{-2}\right]^{\wedge} \\
& =(2 \pi)^{-n} \frac{2 \pi^{n / 2}}{\Gamma(n / 2)}(-1)^{\frac{n-2}{2}} \Delta_{\theta}^{\frac{n-2}{2}}\left[|\theta|^{-1}(\pi-\arccos s) \sqrt{1-s^{2}}\right]
\end{aligned}
$$

for even dimensions $n$, and

$$
\begin{aligned}
& {\left[\frac{\pi}{2}|(x, \xi)||x|^{-2}+i(x, \xi) \ln \left|\left(\frac{x}{|x|}, \xi\right)\right||x|^{-2}\right]^{\wedge}} \\
& \quad=(2 \pi)^{-n} \frac{\pi^{\frac{n+1}{2}}}{\Gamma((n+1) / 2)}(-1)^{\frac{n+1}{2}} \Delta_{\theta}^{\frac{n-1}{2}}[\ln (1-s)-s \ln (1-s)+\ln |\theta|]
\end{aligned}
$$

for odd dimensions.

Our next objective is to show that the above distributional Laplacians are Laplacians in the classical sense and that the resultant functions are integrable on $S^{n-1}$.

Note, that for small $t \geq 0$, we have

$$
\sqrt{t} \arcsin \sqrt{t}=\sum_{j=1}^{\infty} \frac{(2 j-2) !}{2^{2 j-2}((j-1) !)^{2}(2 j-1)} t^{j},
$$

which implies that $\sqrt{1-s^{2}} \arcsin \sqrt{1-s^{2}}$ has (one-sided) derivatives of all orders at $s=1$ and $s=-1$.

For $-1 \leq s \leq 0$, we have

$$
\sqrt{1-s^{2}}(\pi-\arccos s)=\sqrt{1-s^{2}} \arcsin \sqrt{1-s^{2}}
$$

and so it has all (one-sided) derivatives at $s=-1$. On the other hand, if $0 \leq s \leq 1$, then

$$
\sqrt{1-s^{2}}(\pi-\arccos s)=\pi \sqrt{1-s^{2}}-\sqrt{1-s^{2}} \arcsin \sqrt{1-s^{2}}
$$

and thus the singularities of the derivatives of $\sqrt{1-s^{2}}(\pi-\arccos s)$ at $s=1$ are exactly those of $\sqrt{1-s}$.

It is easy to see that, for a sufficiently smooth function $\phi$ of the single variable $s$, we have

$$
\begin{aligned}
& \Delta_{\theta}\left(|\theta|^{-m} \phi(s)\right) \\
& \quad=|\theta|^{-m-2}\left(\left(1-s^{2}\right) \phi^{\prime \prime}(s)-(n-1) s \phi^{\prime}(s)-m(n-m-2) \phi(s)\right) .
\end{aligned}
$$


In particular, if $\phi(s)=(1-s)^{\alpha}$, then $\Delta_{\theta}\left(|\theta|^{-m} \phi(s)\right)$ has order $(1-s)^{\alpha-1}$ as $s \rightarrow 1$. Consequently, in even dimensions, the order of our Fourier transforms is $(1-s)^{-(n-3) / 2}($ as $s \rightarrow 1)$ for $\theta \notin \operatorname{span}\{\xi\}$. In odd dimensions, we are applying Laplacians to the function $(1-s) \ln (1-s)$. Now, $\Delta_{\theta}((1-s) \ln (1-s))$ has order $\ln (1-s)$ as $s \rightarrow 1$ and $\Delta_{\theta}^{2}((1-s) \ln (1-s))$ has order $(1-s)^{-1}$ as $s \rightarrow 1$. Consequently, the order of our Fourier transforms in odd dimensions is also $(1-s)^{-(n-3) / 2}$ as $s \rightarrow 1$. It follows that these Fourier transforms are integrable on $S^{n-1}$, for all dimensions $n$.

Thus the classical Laplacians

$$
\Delta_{\theta}^{\frac{n-2}{2}}\left[|\theta|^{-1}(\pi-\arccos s) \sqrt{1-s^{2}}\right]
$$

and

$$
\Delta_{\theta}^{\frac{n-1}{2}}[\ln (1-s)-s \ln (1-s)+\ln |\theta|]
$$

are integrable as functions of $\theta \in S^{n-1}$.

Finally, we show that, for a test function $\phi$ supported away from the origin,

$$
\begin{aligned}
\left\langle\Delta _ { \theta } ^ { \frac { n - 2 } { 2 } } \left[|\theta|^{-1}(\pi-\arccos s)\right.\right. & \left.\left.\sqrt{1-s^{2}}\right], \phi\right\rangle \\
& =\int_{\mathbb{R}^{n}} \Delta_{\theta}^{\frac{n-2}{2}}\left[|\theta|^{-1}(\pi-\arccos s) \sqrt{1-s^{2}}\right] \phi(\theta) d \theta
\end{aligned}
$$

if $n$ is even and

$$
\begin{aligned}
\left\langle\Delta_{\theta}^{\frac{n-1}{2}}[\ln (1-s)-s \ln (1-s)\right. & +\ln |\theta|], \phi\rangle \\
& =\int_{\mathbb{R}^{n}} \Delta_{\theta}^{\frac{n-1}{2}}[\ln (1-s)-s \ln (1-s)+\ln |\theta|] \phi(\theta) d \theta
\end{aligned}
$$

if $n$ is odd.

We first note that the homogeneity of the distributions is $-n+1$ and so the assumption that $\phi$ is supported away from the origin is justified. We can further assume that $\phi$ has compact support since such functions are dense amongst test functions.

For even $n \geq 2$ and $m=2, \ldots, n$ we put

$$
G_{m}(\theta)=\Delta_{\theta}^{\frac{m-2}{2}}\left[|\theta|^{-1}(\pi-\arccos s) \sqrt{1-s^{2}}\right]
$$

for odd $n \geq 3$ and $m=1,3, \ldots, n$ we put

$$
G_{m}(\theta)=\Delta_{\theta}^{\frac{m-1}{2}}[\ln (1-s)-s \ln (1-s)+\ln |\theta|] .
$$

It will suffice to prove that, for all test functions $\phi$, compactly supported away from the origin,

$$
\left\langle G_{m}, \phi\right\rangle=\int_{\mathbb{R}^{n}} G_{m}(\theta) \phi(\theta) d \theta
$$

for all $m \leq n$. The cases $m=1,2$ are clear and so we may proceed by induction. 
We have, for $m+2 \leq n$,

$$
\left\langle G_{m+2}, \phi\right\rangle=\left\langle\Delta G_{m}, \phi\right\rangle=\left\langle G_{m}, \Delta \phi\right\rangle=\int_{\mathbb{R}^{n}} G_{m}(\theta) \Delta \phi(\theta) d \theta .
$$

We let $A$ be an annulus containing the support of $\phi$. For a small $\epsilon>0$, denote by $C_{\epsilon}$ the full-dimensional cone

$$
C_{\epsilon}=\left\{\theta \in \mathbb{R}^{n}:|(\theta /|\theta|, \xi)| \geq \cos \epsilon\right\} .
$$

We have seen that $\left(1-s^{2}\right)^{(n-3) / 2} G_{m}(\theta)$ has a removable singularity for $\theta \in \operatorname{span}\{\xi\}$ and thus $G_{m}$ is integrable on $A$ if $m \leq n$. It follows from Green's formula that

$$
\begin{aligned}
\int_{\mathbb{R}^{n}} G_{m}(\theta) \Delta \phi(\theta) d \theta=\int_{A} G_{m}(\theta) \Delta \phi(\theta) d \theta=\lim _{\epsilon \rightarrow 0} \int_{A \backslash C_{\epsilon}} G_{m}(\theta) \Delta \phi(\theta) d \theta \\
=\lim _{\epsilon \rightarrow 0}\left[\int_{A \backslash C_{\epsilon}} \Delta G_{m}(\theta) \phi(\theta) d \theta+\int_{\partial\left(A \backslash C_{\epsilon}\right)}\left(G_{m} \frac{\partial \phi}{\partial \nu}-\phi \frac{\partial G_{m}}{\partial \nu}\right) d S\right] \\
=\lim _{\epsilon \rightarrow 0}\left[\int_{A \backslash C_{\epsilon}} G_{m+2}(\theta) \phi(\theta) d \theta+\int_{\partial\left(A \backslash C_{\epsilon}\right)}\left(G_{m} \frac{\partial \phi}{\partial \nu}-\phi \frac{\partial G_{m}}{\partial \nu}\right) d S\right],
\end{aligned}
$$

where $\nu$ denotes the outer unit normal to $\partial\left(A \backslash C_{\epsilon}\right)$. As we have already noted, $G_{m}$ has order $(1-s)^{-(m-3) / 2}$. Consequently, the dominant term in $\nabla G_{m}$ is

$$
(1-s)^{-(m-1) / 2}\left(\frac{1}{|\theta|} \xi-\frac{(\theta, \xi)}{|\theta|^{3}} \theta\right) .
$$

The normal $\nu$ in Green's formula is

$$
\nu=\sin \epsilon \xi-\cos \epsilon \zeta
$$

where $\zeta \in S^{n-1} \cap \xi^{\perp}$. Therefore, on $\partial C_{\epsilon}$,

$$
\frac{\partial G_{m}}{\partial \nu} \sim|\theta|^{-m}(\sin \epsilon)^{-(m-2)}
$$

The boundary $\partial C_{\epsilon}$ is parameterized by the mapping

$$
(t, \zeta) \mapsto t(\cos \epsilon \xi+\sin \epsilon \zeta) \quad(t, \zeta) \in \mathbb{R} \times\left(S^{n-1} \cap \xi^{\perp}\right),
$$

and so its surface area element is given by

$$
d S=(|t| \sin \epsilon)^{n-2} d t d \zeta .
$$

It follows that

$$
\left|\int_{\partial\left(A \backslash C_{\epsilon}\right)}\left(G \frac{\partial \phi}{\partial \nu}-\phi \frac{\partial G}{\partial \nu}\right) d S\right| \leq C \int_{a}^{b} \int_{S^{n-1} \cap \xi^{\perp}}|t|^{n-m-2}(\sin \epsilon)^{n-m} d \zeta d t,
$$

which approaches zero, as $\epsilon \rightarrow 0$, since $C$ depends only on $\max \{\phi, \partial \phi / \partial n\}$ and since $m+2 \leq n$. Thus, we have proved (19) for all $m \leq n$, as required.

The next step is to justify a Parseval formula for (17). Our proof will be based on the use of spherical harmonics. Recall that a spherical harmonic of degree $m$ is the restriction to the sphere of a harmonic homogeneous polynomial of degree $m$. The Hilbert space $L_{2}\left(S^{n-1}\right)$ of real-valued square integrable functions with the usual scalar product $(f, g)=\int_{S^{n-1}} f(x) g(x) d x$ has an orthonormal basis consisting of spherical harmonics, and spherical harmonics of different degrees are mutually 
orthogonal. We say that $\sum_{m=0}^{\infty} F_{m}$ is a spherical harmonic expansion of $f$ when $F_{m}$ is the orthogonal projection of $f$ onto the subspace of spherical harmonics of order $m$. The spherical harmonics of a given degree form an irreducible invariant subspace of $L_{2}\left(S^{n-1}\right)$ with respect to the action of the rotation group. It is, therefore, a consequence of Schur's Lemma that spherical harmonics comprise the eigenspaces of operators on $L^{2}\left(S^{n-1}\right)$ that intertwine this group action. For background information on spherical harmonics, we refer the reader to the book by Groemer [17.

Let us consider the mapping $I_{p}: C^{\infty}\left(S^{n-1}\right) \rightarrow C^{\infty}\left(S^{n-1}\right)$ given by $f \mapsto$ $\left.\hat{f}_{p}\right|_{S^{n-1}}$. It was shown in [15] that this mapping is well defined and that it is injective for $-1 \leq p<n$. It was also shown that, for these $p$, the eigenvectors of $I_{p}$ are the spaces of spherical harmonics of degrees $m=0,1, \ldots$ and the corresponding eigenvalues are

$$
\lambda_{m}(n, p)=\frac{2^{p} \pi^{n / 2}(-1)^{m / 2} \Gamma((m+p) / 2)}{\Gamma((m+n-p) / 2)}
$$

for $(m, p) \neq(0,0),(1,-1)$.

Theorem 5.1. For $\xi \in S^{n-1}$ and $f \in C^{\infty}\left(S^{n-1}\right)$ satisfying (11), we have

$$
\int_{S^{n-1}}|(x, \xi)||x|^{-2} \hat{f}_{n-1}(x) d x=\int_{S^{n-1}}\left(|(x, \xi)||x|^{-2}\right)^{\wedge}(\theta) f(\theta) d \theta
$$

and

$$
\begin{aligned}
\int_{S^{n-1}} \ln |(x, \xi)|(x, \xi)|x|^{-2} \hat{f}_{n-1}(x) & d x \\
= & \int_{S^{n-1}}\left(\ln |(x /|x|, \xi)|(x, \xi)|x|^{-2}\right)^{\wedge}(\theta) f(\theta) d \theta .
\end{aligned}
$$

Proof. We remark that our previous observations establish the integrability of the above Fourier transforms. For infinitely smooth even functions, the spherical Parseval formula of the theorem was proved by Koldobsky; see [24, Lemma 3.22], for example. Another proof was given by E. Milman [25]. We will adapt the latter to cover the odd case.

Let $f$ and $g$ be infinitely smooth functions on the sphere, whose spherical harmonic expansions are

$$
\sum_{m=0}^{\infty} F_{m} \quad \text { and } \quad \sum_{m=0}^{\infty} G_{m}
$$

respectively. Then, when restricted to $S^{n-1}$, the Fourier transforms of the homogeneous extensions $f_{p}$ and $g_{p}, 0<p<n$, have spherical harmonic expansions

$$
\sum_{m=0}^{\infty} \lambda_{m}(n, p) F_{m} \quad \text { and } \quad \sum_{m=0}^{\infty} \lambda_{m}(n, p) G_{m}
$$

for the multipliers $\lambda_{m}(n, p)$ given in (20). It follows that the mapping $I_{p}: C^{\infty}\left(S^{n-1}\right)$ $\rightarrow C^{\infty}\left(S^{n-1}\right)$ given by $f \mapsto \hat{f}_{p}$ is self-adjoint, and so

$$
\int_{S^{n-1}} f(\theta) \hat{g_{p}}(\theta) d \theta=\int_{S^{n-1}} \hat{f}_{p}(\theta) g(\theta) d \theta .
$$

We will use the case $p=n-1$. 
The mapping

$$
J_{1}: f \mapsto \int_{S^{n-1}}|(x, \cdot)||x|^{-2} \hat{f}_{n-1}(x) d x,
$$

restricted to the space of spherical harmonics of degree $m$ in dimension $n$, intertwines the action of the rotation group and therefore acts as a multiple of the identity. Using (2) and the comments above, we see that this multiple is zero for odd $m$ and

$$
-\frac{2}{\pi} \lambda_{m}(n,-1) \lambda_{m}(n, n-1)=-\frac{2^{n+1} \pi^{n-1}}{(m-1)(m+n-1)}
$$

for even values of $m$.

Consequently, $J_{1}$ can be extended to a continuous linear mapping of $C^{\infty}\left(S^{n-1}\right)$ to itself. We now compare it with the mapping $J_{2}: C^{\infty}\left(S^{n-1}\right) \rightarrow C^{\infty}\left(S^{n-1}\right)$ given by

$$
J_{2}: f \mapsto \int_{S^{n-1}}\left(|(x, \cdot)||x|^{-2}\right)^{\wedge}(\theta) f(\theta) d \theta .
$$

Using (2) and (20), together with the addition theorem for spherical harmonics (see [17, Theorem 3.3.3], for example), the spherical harmonic expansion of $|(x, \xi)|$ is

$$
-\frac{2}{\pi n \kappa_{n}} \sum_{\substack{m=0 \\ m \text { even }}}^{\infty} N(n, m) \lambda_{m}(n,-1) P_{m}^{n}((x, \xi)),
$$

where $\kappa_{n}=\pi^{n / 2} / \Gamma(1+n / 2)$ is the volume of the unit ball in $\mathbb{R}^{n}, N(n, m) \simeq m^{n-2}$ is the dimension of the space of spherical harmonics of degree $m$, and $P_{m}^{n}$ denotes the $n$-dimensional Legendre polynomial of degree $m$. Thus $\left(|(x, \xi)||x|^{-2}\right)^{\wedge}$ has spherical harmonic expansion

$$
-\frac{2}{\pi n \kappa_{n}} \sum_{\substack{m=0 \\ m \text { even }}}^{\infty} N(n, m) \lambda_{m}(n,-1) \lambda_{m}(n, n-1) P_{m}^{n}((x, \xi)) ;
$$

compare with equation (3) of [4, page 25]. The addition theorem now shows that $J_{1}$ and $J_{2}$ have the same eigenvalues on any given space of spherical harmonics. Thus, we may write

$$
\int_{S^{n-1}}|(x, \xi)||x|^{-2} \hat{f}_{n-1}(x) d x=\int_{S^{n-1}}\left(|(x, \xi)||x|^{-2}\right)^{\wedge}(\theta) f(\theta) d \theta,
$$

for all $f \in C^{\infty}\left(S^{n-1}\right)$.

The argument for odd functions is similar. The mapping

$$
f \mapsto \int_{S^{n-1}}(x, \cdot)|x|^{-2} \ln |(x, \cdot)| \hat{f}_{n-1}(x) d x,
$$

restricted to the space of spherical harmonics of degree $m$ in dimension $n$, intertwines the action of the rotation group and therefore acts as a multiple of the identity. Using (2) and the comments above, we see that this multiple is zero for even $m$ and

$$
-\lambda_{m}(n,-1) \lambda_{m}(n, n-1)=\frac{2^{n} \pi^{n}}{(m-1)(m+n-1)}
$$

for odd values of $m \neq 1$. For $m \neq 1$, these are also the eigenvalues of the mapping

$$
f \mapsto \int_{S^{n-1}}\left(\ln |(x /|x|, \cdot)|(x, \cdot)|x|^{-2}\right)^{\wedge}(\theta) f(\theta) d \theta .
$$


Thus we may write

$$
\int_{S^{n-1}} \ln |(x, \xi)|(x, \xi)|x|^{-2} \hat{f}_{n-1}(x) d x=\int_{S^{n-1}}\left(\ln |(x /|x|, \xi)|(x, \xi)|x|^{-2}\right)^{\wedge}(\theta) f(\theta) d \theta
$$

for all $f \in C^{\infty}\left(S^{n-1}\right)$ satisfying (11).

Combining Theorem 5.1 with equation (17), we see that $f \in C^{\infty}\left(S^{n-1}\right)$ is the first surface area function of a smooth convex body in $\mathbb{R}^{n}$ if and only if

$$
h(\xi)=\int_{S^{n-1}} g_{n}((\theta, \xi)) f(\theta) d \theta \quad\left(\xi \in S^{n-1}\right)
$$

is a support function, where, for $\theta \in \mathbb{R}^{n} \backslash \operatorname{span}\{\xi\}$ and $s=(\theta /|\theta|, \xi)$,

$$
g_{n}((\theta, \xi))=(2 \pi)^{-n} \frac{2 \pi^{n / 2}}{\Gamma(n / 2)}(-1)^{\frac{n-2}{2}} \Delta_{\theta}^{\frac{n-2}{2}}\left[|\theta|^{-1}(\pi-\arccos s) \sqrt{1-s^{2}}\right]
$$

if $n$ is even and

$$
g_{n}((\theta, \xi))=(2 \pi)^{-n} \frac{(-1)^{\frac{n+1}{2}} \pi^{\frac{n+1}{2}}}{\Gamma((n+1) / 2)} \Delta_{\theta}^{\frac{n-1}{2}}[\ln (1-s)-s \ln (1-s)+\ln |\theta|]
$$

if $n$ is odd.

We will conclude by indicating how this result extends to arbitrary bodies. Instead of dealing with radii of curvature, we must use the first surface area measure. Let $\mu$ be the first area measure of a convex body $K$. The integrability of $g_{n}((\cdot, \xi))$ and Fubini's Theorem show that

$$
\int_{S^{n-1}} \int_{S^{n-1}} g_{n}((\theta, \xi)) \mu(d \theta) d \xi=\int_{S^{n-1}} \int_{S^{n-1}} g_{n}((\theta, \xi)) d \xi \mu(d \theta)
$$

which is finite, and so $\int_{S^{n-1}} g_{n}((\theta, \xi)) \mu(d \theta)$ is finite for almost all $\xi \in S^{n-1}$.

As distributions on the sphere, we have

$$
\square h=\mu,
$$

where $\square=\frac{1}{n-1} \Delta_{S}+1$ is the so-called block operator and $\Delta_{S}$ is the LaplaceBeltrami operator on the sphere. Note that the equation above is an equivalent form of (10).

For any infinitely differentiable function $\phi$ on the sphere we have

$$
\langle\square h, \phi\rangle=\langle\mu, \phi\rangle, \quad \text { that is, }\langle h, \square \phi\rangle=\langle\mu, \phi\rangle .
$$

Since we explained above how to solve equation (10) for infinitely smooth functions, the latter distributional equation implies that for almost all $\xi$,

$$
h_{K}(\xi)=\int_{S^{n-1}} g_{n}((\theta, \xi)) \mu(d \theta),
$$

assuming that the Steiner point of $K$ is at the origin. We note that the proof is now complete in the case that $\mu$ has a continuous density on $S^{n-1}$.

To prove this equality for all $\xi \in S^{n-1}$ and for an arbitrary first surface area measure $\mu$, we refer the reader to [4. p. 49], where Berg shows that two subharmonic functions on the sphere which are equal almost everywhere must be equal everywhere. 


\section{REgularity}

We consider the mapping

$$
f \mapsto \int_{S^{n-1}} g_{n}((\theta, \cdot)) f(\theta) d \theta
$$

and aim to show that it maps $C^{m, \alpha}\left(S^{n-1}\right)$ into $C^{m+2, \beta}\left(S^{n-1}\right)$ for all $0<\beta<$ $\alpha<1$ and $m=0,1, \ldots$. This will be achieved by adapting the classical Euclidean techniques (see, for example, [12, Chap. 4]) to our spherical setting.

Theorem 6.1. Let $f \in C^{m, \alpha}\left(S^{n-1}\right), m \geq 0,0<\alpha<1$, and define the function $h$ by the formula

$$
h(\xi)=\int_{S^{n-1}} g_{n}((\theta, \xi)) f(\theta) d \theta, \quad \xi \in S^{n-1} .
$$

Then $h \in C^{m+2, \beta}\left(S^{n-1}\right)$ for all $0<\beta<\alpha$.

Proof. For the reader's convenience we will first outline the main steps of the proof.

Case 1 . First we deal with the case $m=0$. Choose $1 \leq i, j \leq n$. Our goal is to show that

$$
D_{i, j} h(\xi)=\int_{S^{n-1}} D_{i, j}\left(|\xi| g_{n}((\theta, \xi /|\xi|))\right)(f(\theta)-f(\xi)) d \theta+f(\xi) D_{i, j}|\xi|,
$$

for $f \in C^{\alpha}\left(S^{n-1}\right)$, and then establish the Hölder continuity of the integral in (23).

Step 1. We show the convergence of the integral on the right-hand side of (23) by studying the integrability properties of the function $g_{n}$ and its derivatives.

Step 2. Since $g_{n}(s)$ has a singularity at $s=1$, we consider a sequence of smooth functions that approximate $g_{n}$. Then we differentiate under the integral sign in (22) and, after passing to the limit, we show that

$$
D_{i} h(\xi)=\int_{S^{n-1}} D_{i}\left(|\xi| g_{n}((\theta, \xi /|\xi|))\right) f(\theta) d \theta .
$$

Step 3. Using a differential equation for $g_{n}$, established by Berg, the same ideas as in Step 2 allow us to compute the second derivative and prove (23).

Step 4. A more detailed analysis of (23) yields the required Hölder regularity.

Case 2. $m>0$.

Step 1. We show that an integral representation, similar to (22), exists for higher order derivatives of $h$, namely

$$
D^{m} h(\xi)=\int_{S^{n-1}} g_{m, n}((\theta, \xi)) D^{m} f(\theta) d \theta .
$$

Step 2. It turns out that the functions $g_{m, n}(s)$ and their first and second derivatives have the same order of magnitude as $g_{n}(s)$ and its derivatives. Therefore, applying Steps 1 through 4 from Case 1 yields the Hölder regularity of $D^{m} h$. The only detail that has to be checked is whether the functions $g_{m, n}(s)$ satisfy a certain differential equation.

Now we will carry out a detailed proof of each of these steps. 
Case 1. Step 1. The convergence of the integral in (23), as well as its Hölder continuity, will follow from our estimates on the behavior of $g_{n}(s)$ and its derivatives at $s=1$. The proof of equation (23) will make use of a differential equation satisfied by $g_{n}$ on $(-1,1)($ see $(26)$ ), as well as the above estimates.

First, we study the integrability properties of $D_{i, j}\left(|\xi| g_{n}((\theta, \xi /|\xi|))\right)$ on the sphere. One sees that

$$
\begin{aligned}
\left.D_{i, j}\left(|\xi| g_{n}((\theta, \xi /|\xi|))\right)\right|_{|\xi|=1}=\left(\delta_{i j}\right. & \left.-\xi_{i} \xi_{j}\right)\left(g_{n}((\theta, \xi))-(\theta, \xi) g_{n}^{\prime}((\theta, \xi))\right) \\
& +\left(\theta_{i}-(\theta, \xi) \xi_{i}\right)\left(\theta_{j}-(\theta, \xi) \xi_{j}\right) g_{n}^{\prime \prime}((\theta, \xi))
\end{aligned}
$$

We recall that the dominant term in $g_{n}(s)$ is of the form $(1-s)^{-(n-3) / 2}$. Consequently $g_{n}^{\prime}(s)$ and $g_{n}^{\prime \prime}(s)$ have dominant terms $(1-s)^{-(n-1) / 2}$ and $(1-s)^{-(n+1) / 2}$, respectively. We will also use the fact that, for $f \in C^{\alpha}\left(S^{n-1}\right)$, there is a constant $[f]_{\alpha}$ such that

$$
|f(\theta)-f(\xi)| \leq[f]_{\alpha}|\theta-\xi|^{\alpha}=2^{\alpha / 2}[f]_{\alpha}(1-(\theta, \xi))^{\alpha / 2}
$$

for all $\theta, \xi \in S^{n-1}$.

We again use cylindrical coordinates to estimate the integral term in (23). Put

$$
\theta=s \xi+\sqrt{1-s^{2}} \zeta, \quad \text { where } \zeta \in \xi^{\perp} \cap S^{n-1} .
$$

With this notation,

$$
\theta_{i}-(\theta, \xi) \xi_{i}=\sqrt{1-s^{2}} \zeta_{i}
$$

Here, and in the sequel, $C$ will denote a (variable positive) constant which depends only on $f, \alpha, i, j, n$. We have

$$
\begin{aligned}
& \left|\int_{S^{n-1}} D_{i, j}\left(|\xi| g_{n}((\theta, \xi /|\xi|))\right)(f(\theta)-f(\xi)) d \theta\right| \\
& \leq C \int_{-1}^{1}(1-s)^{(n-3+\alpha) / 2} \mid \int_{S^{n-1} \cap \xi^{\perp}}\left[\left(\delta_{i j}-\xi_{i} \xi_{j}\right)\left(g_{n}(s)-s g_{n}^{\prime}(s)\right)\right. \\
& \left.+\left(1-s^{2}\right) g_{n}^{\prime \prime}(s) \zeta_{i} \zeta_{j}\right] d \zeta \mid d s \\
& \leq C \int_{-1}^{1}(1-s)^{-1+\alpha / 2} d s<\infty
\end{aligned}
$$

since $\alpha>0$. Therefore, the integral in (23) is convergent.

Step 2. In order to prove formula (23), we first want to show

$$
D_{i} h(\xi)=\int_{S^{n-1}} D_{i}\left(|\xi| g_{n}((\theta, \xi /|\xi|))\right) f(\theta) d \theta
$$

Let $\eta:[0, \infty) \rightarrow[0,1]$ be a $C^{\infty}$-function with $\eta=0$ on $[0,1], \eta=1$ on $[2, \infty)$ and $0 \leq \eta^{\prime} \leq 2$. We put, for $\varepsilon>0$ and $\xi \in S^{n-1}$,

$$
h_{\varepsilon}(\xi)=\int_{S^{n-1}} \eta((1-(\theta, \xi)) / \varepsilon) g_{n}((\theta, \xi)) f(\theta) d \theta \text {. }
$$

We see that $h_{\varepsilon}(\xi) \rightarrow h(\xi)$ as $\varepsilon \rightarrow 0$ uniformly in $\xi \in S^{n-1}$. To prove (25) it is enough to show that $D_{i} h_{\varepsilon}(\xi)$ converges uniformly to the right-hand side of (25) as 
$\varepsilon \rightarrow 0$. We have

$$
\begin{aligned}
D_{i} h_{\varepsilon}(\xi)= & \int_{S^{n-1}} D_{i}\left[\eta((1-(\theta, \xi /|\xi|)) / \varepsilon)|\xi| g_{n}((\theta, \xi /|\xi|))\right] f(\theta) d \theta \\
= & \int_{S^{n-1}} D_{i}[\eta((1-(\theta, \xi /|\xi|)) / \varepsilon)]|\xi| g_{n}((\theta, \xi /|\xi|)) f(\theta) d \theta \\
& \quad+\int_{S^{n-1}} \eta((1-(\theta, \xi)) / \varepsilon) D_{i}\left[|\xi| g_{n}((\theta, \xi /|\xi|))\right] f(\theta) d \theta .
\end{aligned}
$$

The second integral uniformly approaches the right-hand side of (25), and so it is enough to show that the first integral converges uniformly to zero. We have

$$
\begin{aligned}
\mid \int_{S^{n-1}} & D_{i}[\eta((1-(\theta, \xi /|\xi|)) / \varepsilon)] g_{n}((\theta, \xi)) f(\theta) d \theta \mid \\
= & \frac{1}{\varepsilon}\left|\int_{S^{n-1}}\left((\theta, \xi) \xi_{i}-\theta_{i}\right) \eta^{\prime}((1-(\theta, \xi)) / \varepsilon) g_{n}((\theta, \xi)) f(\theta) d \theta\right| \\
= & \frac{1}{\varepsilon} \mid \int_{1-2 \varepsilon}^{1-\varepsilon}\left(1-s^{2}\right)^{(n-3) / 2} \sqrt{1-s^{2}} \eta^{\prime}((1-s) / \varepsilon) g_{n}(s) \\
& \times \int_{S^{n-1} \cap \xi^{\perp}} \zeta_{i} f\left(s \xi+\sqrt{1-s^{2}} \zeta\right) d \zeta d s \mid \\
\leq & \frac{C}{\varepsilon} \int_{1-2 \varepsilon}^{1-\varepsilon}(1-s)^{(n-2) / 2}(1-s)^{-(n-3) / 2} d s \\
= & \left.\frac{C}{\varepsilon}(1-s)^{3 / 2}\right|_{1-\varepsilon} ^{1-2 \varepsilon}=C \varepsilon^{1 / 2} .
\end{aligned}
$$

Step 3. Now we will prove (23). For $\varepsilon>0$, we set

$$
v_{\varepsilon}(\xi)=\int_{S^{n-1}} \eta((1-(\theta, \xi /|\xi|)) / \varepsilon) D_{i}\left(|\xi| g_{n}((\theta, \xi /|\xi|))\right) f(\theta) d \theta .
$$

Then $v_{\varepsilon} \rightarrow D_{i} h$ uniformly as $\varepsilon \rightarrow 0$. To prove (23) it suffices to show that $D_{j} v_{\varepsilon}$ uniformly converges to the right-hand side of (23) as $\varepsilon \rightarrow 0$. We have

$$
\begin{aligned}
& D_{j} v_{\varepsilon}(\xi) \\
& =\int_{S^{n-1}} \eta((1-(\theta, \xi /|\xi|)) / \varepsilon) D_{i, j}\left(|\xi| g_{n}((\theta, \xi /|\xi|))\right)(f(\theta)-f(\xi)) d \theta \\
& \quad+f(\xi) \int_{S^{n-1}} \eta((1-(\theta, \xi /|\xi|)) / \varepsilon) D_{i, j}\left(|\xi| g_{n}((\theta, \xi /|\xi|))\right) d \theta \\
& \quad+\int_{S^{n-1}} D_{j}[\eta((1-(\theta, \xi /|\xi|)) / \varepsilon)] D_{i}\left(|\xi| g_{n}((\theta, \xi /|\xi|))\right)(f(\theta)-f(\xi)) d \theta \\
& \quad+f(\xi) \int_{S^{n-1}} D_{j}[\eta((1-(\theta, \xi /|\xi|)) / \varepsilon)] D_{i}\left(|\xi| g_{n}((\theta, \xi /|\xi|))\right) d \theta \\
& \quad=I_{1}+I_{2}+I_{3}+I_{4} .
\end{aligned}
$$

We see that $I_{1}$ converges uniformly to the integral term in (23). Our goal is to show that $I_{2}$ and $I_{3}$ approach zero uniformly and also that the limit of $I_{4}$ is $f(\xi)\left(\delta_{i j}-\xi_{i} \xi_{j}\right)$, which is exactly what we need. 
For $I_{2}$ we use formula (24) and cylindrical coordinates to get

$$
\begin{aligned}
& \int_{S^{n-1}} \eta((1-(\theta, \xi)) / \varepsilon) D_{i, j}\left(|\xi| g_{n}((\theta, \xi /|\xi|))\right) d \theta \\
& \begin{array}{l}
=\int_{S^{n-1}} \eta((1-(\theta, \xi)) / \varepsilon)\left(\delta_{i j}-\xi_{i} \xi_{j}\right)\left(g_{n}((\theta, \xi))-(\theta, \xi) g_{n}^{\prime}((\theta, \xi))\right) d \theta \\
\quad+\int_{S^{n-1}} \eta((1-(\theta, \xi)) / \varepsilon)\left(\theta_{i}-(\theta, \xi) \xi_{i}\right)\left(\theta_{j}-(\theta, \xi) \xi_{j}\right) g_{n}^{\prime \prime}((\theta, \xi)) d \theta \\
=(n-1) \kappa_{n-1}\left(\delta_{i j}-\xi_{i} \xi_{j}\right) \\
\quad \times \int_{-1}^{1} \eta((1-s) / \varepsilon)\left(1-s^{2}\right)^{(n-3) / 2}\left(g_{n}(s)-s g_{n}^{\prime}(s)\right) d s \\
\quad+\int_{-1}^{1} \eta((1-s) / \varepsilon)\left(1-s^{2}\right)^{(n-1) / 2} g_{n}^{\prime \prime}(s) \int_{\xi^{\perp} \cap S^{n-1}} \zeta_{i} \zeta_{j} d \zeta d s .
\end{array}
\end{aligned}
$$
$b$,

We claim that $\int_{\xi^{\perp} \cap S^{n-1}} \zeta_{i} \zeta_{j} d \zeta=\kappa_{n-1}\left(\delta_{i j}-\xi_{i} \xi_{j}\right)$. Indeed, for any vectors $a$ and

$$
\begin{aligned}
\int_{S^{n-1}}(a, u)(b, u) d u=a_{1} b_{1} \int_{S^{n-1}} & u_{1}^{2} d u+\cdots+a_{n} b_{n} \int_{S^{n-1}} u_{n}^{2} d u \\
& =(a, b) \frac{1}{n} \int_{S^{n-1}}\left(u_{1}^{2}+\cdots+u_{n}^{2}\right) d u=\kappa_{n}(a, b) .
\end{aligned}
$$

Therefore, denoting by $e_{i} \mid \xi^{\perp}$ and $e_{j} \mid \xi^{\perp}$ the orthogonal projections of $e_{i}$ and $e_{j}$ onto the subspace $\xi^{\perp}$, we have

$$
\begin{aligned}
\int_{\xi^{\perp} \cap S^{n-1}} \zeta_{i} \zeta_{j} d \zeta=\int_{\xi^{\perp} \cap S^{n-1}}\left(e_{i} \mid \xi^{\perp}, \zeta\right) & \left(e_{j} \mid \xi^{\perp}, \zeta\right) d \zeta \\
= & \kappa_{n-1}\left(e_{i}\left|\xi^{\perp}, e_{j}\right| \xi^{\perp}\right)=\kappa_{n-1}\left(\delta_{i j}-\xi_{i} \xi_{j}\right),
\end{aligned}
$$

since $e_{i} \mid \xi^{\perp}=e_{i}-\xi_{i} \xi$ and $e_{j} \mid \xi^{\perp}=e_{j}-\xi_{j} \xi$. It follows that

$$
\begin{aligned}
& \int_{S^{n-1}} \eta((1-(\theta, \xi)) / \varepsilon) D_{i, j}\left(|\xi| g_{n}((\theta, \xi /|\xi|))\right) d \theta \\
& =\kappa_{n-1}\left(\delta_{i j}-\xi_{i} \xi_{j}\right) \int_{-1}^{1} \eta((1-s) / \varepsilon)\left(1-s^{2}\right)^{(n-3) / 2} \\
& \quad \times\left((n-1) g_{n}(s)-(n-1) s g_{n}^{\prime}(s)+\left(1-s^{2}\right) g_{n}^{\prime \prime}(s)\right) d s \\
& =c\left(\delta_{i j}-\xi_{i} \xi_{j}\right) \int_{-1}^{1} \eta((1-s) / \varepsilon) s\left(1-s^{2}\right)^{(n-3) / 2} d s \\
& \rightarrow c\left(\delta_{i j}-\xi_{i} \xi_{j}\right) \int_{-1}^{1} s\left(1-s^{2}\right)^{(n-3) / 2} d s=0 \quad(\text { as } \varepsilon \rightarrow 0),
\end{aligned}
$$

where we used the fact that, for $s \in(-1,1)$,

$$
(n-1) g_{n}(s)-(n-1) s g_{n}^{\prime}(s)+\left(1-s^{2}\right) g_{n}^{\prime \prime}(s)=c s
$$


cf. [4. p. 36]. In Berg's paper the latter constant $c$ is given explicitly. However, its value is clearly irrelevant here, and, furthermore, the constant may be different in our case since, as we mentioned in the introduction, we ignored linear terms while computing $g_{n}$. We are not giving a proof of the above formula at this stage, but the reader will see a proof of a more general formula (see (30) towards the end of this section.

Now we study $I_{3}$ :

$$
\begin{aligned}
\mid \int_{S^{n-1}} D_{j}[ & \eta((1-(\theta, \xi /|\xi|)) / \varepsilon)] D_{i}\left(|\xi| g_{n}((\theta, \xi /|\xi|))\right)(f(\theta)-f(\xi)) d \theta \mid \\
= & \mid \frac{1}{\varepsilon} \int_{S^{n-1}}\left((\theta, \xi) \xi_{j}-\theta_{j}\right) \eta^{\prime}((1-(\theta, \xi)) / \varepsilon) \\
& \quad \times\left(\xi_{i} g_{n}((\theta, \xi))+\left(\theta_{i}-(\theta, \xi) \xi_{i}\right) g_{n}^{\prime}((\theta, \xi))\right)(f(\theta)-f(\xi)) d \theta \mid \\
\leq & \frac{C}{\varepsilon} \int_{S^{n-1}}\left|(\theta, \xi) \xi_{j}-\theta_{j}\right| \eta^{\prime}((1-(\theta, \xi)) / \varepsilon) \\
& \quad \times\left(\left|g_{n}((\theta, \xi))\right|+\left|\theta_{i}-(\theta, \xi) \xi_{i}\right|\left|g_{n}^{\prime}((\theta, \xi))\right|\right)|\theta-\xi|^{\alpha} d \theta \\
\leq & \frac{C}{\varepsilon} \int_{1-2 \varepsilon}^{1-\varepsilon}\left(1-s^{2}\right)^{(n-3) / 2} \int_{S^{n-1} \cap \xi^{\perp}} \sqrt{1-s^{2}}\left|\zeta_{j}\right| \\
& \quad \times\left(\left|g_{n}(s)\right|+\sqrt{1-s^{2}}\left|\zeta_{i}\right|\left|g_{n}^{\prime}(s)\right|\right)(1-s)^{\alpha / 2} d \zeta d s \\
\leq & \frac{C}{\varepsilon} \int_{1-2 \varepsilon}^{1-\varepsilon}(1-s)^{(n-3) / 2}(1-s)(1-s)^{-(n-1) / 2}(1-s)^{\alpha / 2} d s \\
= & \left.\frac{C}{\varepsilon}(1-s)^{1+\alpha / 2}\right|_{1-\varepsilon} ^{1-2 \varepsilon}=c \varepsilon^{\alpha / 2} \rightarrow 0 \quad(\text { as } \varepsilon \rightarrow 0) .
\end{aligned}
$$

Finally, for $I_{4}$, we have

$$
\begin{aligned}
\int_{S^{n-1}} D_{j}[ & \eta((1-(\theta, \xi /|\xi|)) / \varepsilon)] D_{i}\left(|\xi| g_{n}((\theta, \xi /|\xi|))\right) d \theta \\
=\frac{1}{\varepsilon} \int_{S^{n-1}}\left((\theta, \xi) \xi_{j}-\theta_{j}\right) \eta^{\prime}((1-(\theta, \xi)) / \varepsilon) & \\
& \quad \times\left(\xi_{i} g_{n}((\theta, \xi))+\left(\theta_{i}-(\theta, \xi) \xi_{i}\right) g_{n}^{\prime}((\theta, \xi))\right) d \theta \\
=-\frac{1}{\varepsilon} & \int_{-1}^{1}\left(1-s^{2}\right)^{(n-3) / 2} \int_{S^{n-1} \cap \xi^{\perp}} \sqrt{1-s^{2}} \zeta_{j} \eta^{\prime}((1-s) / \varepsilon) \\
& \quad \times\left(\xi_{i} g_{n}(s)+\sqrt{1-s^{2}} \zeta_{i} g_{n}^{\prime}(s)\right) d \zeta d s \\
=-\frac{1}{\varepsilon} & \int_{-1}^{1}\left(1-s^{2}\right)^{(n-1) / 2} \eta^{\prime}((1-s) / \varepsilon) g_{n}^{\prime}(s) d s \int_{S^{n-1} \cap \xi^{\perp}} \zeta_{j} \zeta_{i} d \zeta \\
=-\frac{1}{\varepsilon} & \kappa_{n-1}\left(\delta_{i j}-\xi_{i} \xi_{j}\right) \int_{-1}^{1}\left(1-s^{2}\right)^{(n-1) / 2} \eta^{\prime}((1-s) / \varepsilon) g_{n}^{\prime}(s) d s .
\end{aligned}
$$


Integration by parts shows that this integral is

$$
\begin{gathered}
\kappa_{n-1}\left(\delta_{i j}-\xi_{i} \xi_{j}\right)\left(\left.\left(1-s^{2}\right)^{(n-1) / 2} g_{n}^{\prime}(s) \eta((1-s) / \varepsilon)\right|_{-1} ^{1}\right. \\
\left.-\int_{-1}^{1} \eta((1-s) / \varepsilon)\left(1-s^{2}\right)^{(n-3) / 2}\left(\left(1-s^{2}\right) g_{n}^{\prime \prime}(s)-(n-1) s g_{n}^{\prime}(s)\right) d s\right) \\
=\kappa_{n-1}\left(\delta_{i j}-\xi_{i} \xi_{j}\right) \int_{-1}^{1} \eta((1-s) / \varepsilon)\left(1-s^{2}\right)^{(n-3) / 2}\left[(n-1) g_{n}(s)+c s\right] d s \\
\rightarrow_{\varepsilon \rightarrow 0} \kappa_{n-1}\left(\delta_{i j}-\xi_{i} \xi_{j}\right) \int_{-1}^{1}\left(1-s^{2}\right)^{(n-3) / 2}\left[(n-1) g_{n}(s)+c s\right] d s \\
=\left(\delta_{i j}-\xi_{i} \xi_{j}\right) \int_{S^{n-1}} g_{n}((\theta, \xi)) d \theta=\delta_{i j}-\xi_{i} \xi_{j} .
\end{gathered}
$$

Thus, we have established (23).

Step 4. Now we will show that, for $f \in C^{\alpha}\left(S^{n-1}\right)$, the function

$$
\Phi(\xi)=\int_{S^{n-1}} D_{i, j}\left(|\xi| g_{n}((\theta, \xi /|\xi|))\right)(f(\theta)-f(\xi)) d \theta
$$

is of class $C^{\beta}\left(S^{n-1}\right)$ for all $0<\beta<\alpha$. Let $\xi, \bar{\xi} \in S^{n-1}$. Put $\delta=|\xi-\bar{\xi}|$, $N(\xi)=\left\{\theta \in S^{n-1}:|\theta-\xi|<\delta\right\}$ and $N(\bar{\xi})=\left\{\theta \in S^{n-1}:|\theta-\bar{\xi}|<\delta\right\}$. We have

$$
\begin{aligned}
\Phi(\xi)-\Phi(\bar{\xi}) & =\int_{N(\xi)} D_{i, j}\left(|\xi| g_{n}((\theta, \xi /|\xi|))\right)(f(\theta)-f(\xi)) d \theta \\
& -\int_{N(\bar{\xi})} D_{i, j}\left(|\bar{\xi}| g_{n}((\theta, \bar{\xi} /|\bar{\xi}|))\right)(f(\theta)-f(\bar{\xi})) d \theta \\
& +\int_{S^{n-1} \backslash N(\xi)} D_{i, j}\left(|\xi| g_{n}((\theta, \xi /|\xi|))\right)(f(\theta)-f(\xi)) d \theta \\
& -\int_{S^{n-1} \backslash N(\bar{\xi})} D_{i, j}\left(|\bar{\xi}| g_{n}((\theta, \bar{\xi} /|\bar{\xi}|))\right)(f(\theta)-f(\bar{\xi})) d \theta \\
& =\tilde{I}_{1}+\tilde{I}_{2}+\tilde{I}_{3}+\tilde{I}_{4} .
\end{aligned}
$$

For $\tilde{I}_{1}$, we have

$$
\begin{aligned}
\left|\tilde{I}_{1}\right| \leq C \int_{N(\xi)} \mid D_{i, j} & \left(|\xi| g_{n}((\theta, \xi /|\xi|))\right)|| \theta-\left.\xi\right|^{\alpha} d \theta \\
\leq & C\left(\int_{1-\delta^{2} / 2}^{1}\left|g_{n}(s)-s g_{n}^{\prime}(s)\right|(1-s)^{\alpha / 2+(n-3) / 2} d s\right. \\
& \left.\quad+\int_{1-\delta^{2} / 2}^{1}(1-s)^{\alpha / 2+1+(n-3) / 2}\left|g_{n}^{\prime \prime}(s)\right| \int_{\xi^{\perp} \cap S^{n-1}}\left|\zeta_{i} \zeta_{j}\right| d \zeta d s\right) .
\end{aligned}
$$

Using the previous estimates for $g_{n}$ and its derivatives, we get

$$
\left|\tilde{I}_{1}\right| \leq C \int_{1-\delta^{2} / 2}^{1}\left((1-s)^{\alpha / 2}+(1-s)^{\alpha / 2-1}+(1-s)^{\alpha / 2-1}\right) d s \leq C \delta^{\alpha} .
$$

For $\tilde{I}_{2}$, the same calculations yield

$$
\left|\tilde{I}_{2}\right| \leq C \delta^{\alpha} .
$$


For $\tilde{I}_{3}$ and $\tilde{I}_{4}$, we have

$$
\begin{aligned}
\tilde{I}_{3}+\tilde{I}_{4}= & \int_{-1}^{1-\delta^{2} / 2}\left(1-s^{2}\right)^{(n-3) / 2} \int_{\xi^{\perp} \cap S^{n-1}}\left(\left(\delta_{i j}-\xi_{i} \xi_{j}\right)\left(g_{n}(s)-s g_{n}^{\prime}(s)\right)\right. \\
& \left.+g_{n}^{\prime \prime}(s)\left(1-s^{2}\right) \zeta_{i} \zeta_{j}\right)\left(f\left(s \xi+\sqrt{1-s^{2}} \zeta\right)-f(\xi)\right) d \zeta d s \\
- & \int_{-1}^{1-\delta^{2} / 2}\left(1-s^{2}\right)^{(n-3) / 2} \int_{\bar{\xi}^{\perp} \cap S^{n-1}}\left(\left(\delta_{i j}-\bar{\xi}_{i} \bar{\xi}_{j}\right)\left(g_{n}(s)-s g_{n}^{\prime}(s)\right)\right. \\
& \left.+g_{n}^{\prime \prime}(s)\left(1-s^{2}\right) \zeta_{i} \zeta_{j}\right)\left(f\left(s \bar{\xi}+\sqrt{1-s^{2}} \zeta\right)-f(\bar{\xi})\right) d \xi d s \\
= & J_{1}+J_{2},
\end{aligned}
$$

where

$$
\begin{aligned}
& J_{1}=\int_{-1}^{1-\delta^{2} / 2}(1-\left.s^{2}\right)^{(n-3) / 2} \int_{\xi^{\perp} \cap S^{n-1}}\left(\delta_{i j}-\xi_{i} \xi_{j}\right)\left(g_{n}(s)-s g_{n}^{\prime}(s)\right) \\
& \times\left(f\left(s \xi+\sqrt{1-s^{2}} \zeta\right)-f(\xi)\right) d \zeta d s \\
&-\int_{-1}^{1-\delta^{2} / 2}\left(1-s^{2}\right)^{(n-3) / 2} \int_{\bar{\xi}^{\perp} \cap S^{n-1}}\left(\delta_{i j}-\bar{\xi}_{i} \bar{\xi}_{j}\right)\left(g_{n}(s)-s g_{n}^{\prime}(s)\right) \\
& \times\left(f\left(s \bar{\xi}+\sqrt{1-s^{2}} \zeta\right)-f(\bar{\xi})\right) d \zeta d s
\end{aligned}
$$

and

$$
\begin{aligned}
J_{2}=\int_{-1}^{1-\delta^{2} / 2}\left(1-s^{2}\right)^{(n-3) / 2} \int_{\xi^{\perp} \cap S^{n-1}} & g_{n}^{\prime \prime}(s)\left(1-s^{2}\right) \zeta_{i} \zeta_{j} \\
& \times\left(f\left(s \xi+\sqrt{1-s^{2}} \zeta\right)-f(\xi)\right) d \zeta d s \\
-\int_{-1}^{1-\delta^{2} / 2}\left(1-s^{2}\right)^{(n-3) / 2} & \int_{\bar{\xi}^{\perp} \cap S^{n-1}} g_{n}^{\prime \prime}(s)\left(1-s^{2}\right) \zeta_{i} \zeta_{j} \\
& \times\left(f\left(s \bar{\xi}+\sqrt{1-s^{2}} \zeta\right)-f(\bar{\xi})\right) d \zeta d s
\end{aligned}
$$

Let $A \in S O(n)$ be a matrix such that $\bar{\xi}=A \xi$. We can assume that it is close to the identity matrix, more precisely $\|A-I\| \leq \delta$. After a change of variables of integration we have

$$
\begin{aligned}
\int_{\bar{\xi}^{\perp} \cap S^{n-1}}\left(f\left(s \bar{\xi}+\sqrt{1-s^{2}} \zeta\right)-\right. & f(\bar{\xi})) d \zeta \\
& =\int_{\xi^{\perp} \cap S^{n-1}}\left(f\left(s \bar{\xi}+\sqrt{1-s^{2}} A \zeta\right)-f(\bar{\xi})\right) d \zeta
\end{aligned}
$$


Therefore,

$$
\begin{aligned}
\left|J_{1}\right| & \leq\left|\delta_{i j}-\xi_{i} \xi_{j}\right| \int_{-1}^{1-\delta^{2} / 2}\left(1-s^{2}\right)^{(n-3) / 2}\left|g_{n}(s)-s g_{n}^{\prime}(s)\right| \\
& \times \int_{\xi^{\perp} \cap S^{n-1}}\left(\left|f\left(s \xi+\sqrt{1-s^{2}} \zeta\right)-f\left(s \bar{\xi}+\sqrt{1-s^{2}} A \zeta\right)\right|\right. \\
& +|f(\xi)-f(\bar{\xi})|) d \zeta d s \\
& +\left|\bar{\xi}_{i} \bar{\xi}_{j}-\xi_{i} \xi_{j}\right| \int_{-1}^{1-\delta^{2} / 2}\left(1-s^{2}\right)^{(n-3) / 2}\left|g_{n}(s)-s g_{n}^{\prime}(s)\right| \\
& \times \int_{\bar{\xi}^{\perp} \cap S^{n-1}}\left|f\left(s \bar{\xi}+\sqrt{1-s^{2}} \zeta\right)-f(\bar{\xi})\right| d \zeta d s \\
& \leq C \int_{-1}^{1-\delta^{2} / 2}\left(1-s^{2}\right)^{(n-3) / 2}(1-s)^{-(n-1) / 2} \\
& \left.\times\left.\int_{\xi^{\perp} \cap S^{n-1}}\left(\mid s \xi+\sqrt{1-s^{2}} \zeta-s \bar{\xi}-\sqrt{1-s^{2}} A \zeta\right)\right|^{\alpha}+|\xi-\bar{\xi}|^{\alpha}\right) d \zeta d s \\
& \leq C \delta^{\alpha} \ln \delta^{-1},
\end{aligned}
$$

since $|\xi-\bar{\xi}| \leq \delta$ and $|\zeta-A \zeta| \leq \delta$.

Similar computations yield $\left|J_{2}\right| \leq C \delta^{\alpha} \ln \delta^{-1}$. This completes the proof of the theorem for $m=0$.

Case 2. To handle the general case $m=1,2, \ldots$, we will denote by $D^{m}$ the derivative

$$
D^{m}=\frac{\partial^{m}}{\partial \xi_{i_{1}} \cdots \partial \xi_{i_{m}}} .
$$

We aim to show that there is a function $g_{m, n}$ such that, for $f \in C^{m, \alpha}\left(S^{n-1}\right)$ and extended to $\mathbb{R}^{n} \backslash o$ with homogeneity -1 ,

$$
D^{m} h(\xi)=\int_{S^{n-1}} g_{m, n}((\theta, \xi)) D^{m} f(\theta) d \theta .
$$

The required result will then follow (from the above argument in the case $m=0$ ) if we can establish the appropriate properties of the function $g_{m, n}$. Specifically, we will prove that, for fixed $\xi \in \mathbb{R}^{n} \backslash o, g_{m, n}((\theta, \xi))$ is a function of $\theta \in S^{n-1}$ with dominant term of the form $(1-s)^{-(n-3) / 2}$ and that $g_{m, n}$ satisfies a differential equation analogous to (26).

For even values of $m$, we will see that

$$
g_{m, n}((\theta, \xi))=\Delta_{\xi}^{m / 2}\left[\left(|(x, \xi)||x|^{-m-2}\right)^{\wedge}(\theta)+F(\theta, \xi)\right],
$$

where $F \in C^{\infty}\left(\left(\mathbb{R}^{n} \backslash o\right) \times\left(\mathbb{R}^{n} \backslash o\right)\right)$ and, for each $\theta \in S^{n-1}$, the Fourier transform $\left(|(x, \xi)||x|^{-m-2}\right)^{\wedge}(\theta)$ is extended to $\xi \in \mathbb{R}^{n} \backslash o$ with homogeneity 1 . The smoothness of $F$ implies that this part of the integrand will play no role in estimating the behavior of $g_{m, n}(s)$ as $s \rightarrow 1$.

The proof for odd values of $m$, for which we will not give the details, is based on the properties of

$$
\Delta_{\xi}^{(m-1) / 2}\left(\left(\Gamma^{\prime}(1)-\ln |(x /|x|, \xi)|\right)|x|^{-m-1}\right)^{\wedge}(\theta) .
$$


Step 1. We will next prove that (27) holds for $f \in C^{\infty}\left(S^{n-1}\right)$. We recall that

$$
h^{e}(\xi)=-(2 \pi)^{-n}\left(|x|^{-2} \hat{f}^{e}(x)\right)^{\wedge}(\xi) \quad \text { and } \quad h^{o}(\xi)=(2 \pi)^{-n}\left(|x|^{-2} \hat{f}^{o}(x)\right)^{\wedge}(\xi),
$$

if $f$ is extended to $\mathbb{R}^{n}$ with homogeneity -1 ; see (12) and (13). Thus

$$
D^{m} h^{e}(\xi)=-(2 \pi)^{-n}\left(|x|^{-2}\left(D^{m} f^{e}\right)^{\wedge}(x)\right)^{\wedge}(\xi)
$$

and

$$
D^{m} h^{o}(\xi)=(2 \pi)^{-n}\left(|x|^{-2}\left(D^{m} f^{o}\right)^{\wedge}(x)\right)^{\wedge}(\xi) .
$$

We will focus our attention on the even parts of $f$ and $h$; the proof for the odd parts is analogous. Consequently our objective is to compute

$$
\left(|x|^{-2}\left(D^{m} f^{e}\right)^{\wedge}(x)\right)^{\wedge}(\xi) .
$$

As we have done previously, we will make use of the Laplacian and, therefore, first calculate

$$
\left(|x|^{-m-2}\left(D^{m} f^{e}\right)^{\wedge}(x)\right)^{\wedge}(\xi) \quad \text { for } m \text { even }
$$

and

$$
\left(|x|^{-m-1}\left(D^{m} f^{e}\right)^{\wedge}(x)\right)^{\wedge}(\xi) \quad \text { for } m \text { odd }
$$

As mentioned above, we will just give the details for $m$ even, since the odd case is similar. We will, further, consider separately the cases $m \leq n-2$ and $m \geq n-1$.

For $m \leq n-2$, the arguments in the previous section show that $\left(|(x, \xi)||x|^{-m-2}\right)^{\wedge}$ is integrable on $S^{n-1}$, and the proof of Theorem 5.1 also yields the following result:

For $\xi \in S^{n-1}, m \leq n-2$ and $f \in C^{\infty}\left(S^{n-1}\right)$ satisfying (11), we have

$$
\int_{S^{n-1}}|(x, \xi)||x|^{-m-2} \hat{f}_{n-m-1}(x) d x=\int_{S^{n-1}}\left(|(x, \xi)||x|^{-m-2}\right)^{\wedge}(\theta) f(\theta) d \theta .
$$

We note that, since $m$ is even, $D^{m} f^{e}$ is also even and $|x|^{-m-2}\left(D^{m} f^{e}\right)^{\wedge}(x)$ has homogeneity $-n-1$. Thus (2) and (29) give

$$
\begin{aligned}
\left(|x|^{-m-2}\left(D^{m} f^{e}\right)^{\wedge}(x)\right)^{\wedge}(\xi) & =-\frac{\pi}{2} \int_{S^{n-1}}|(x, \xi)||x|^{-m-2}\left(D^{m} f^{e}\right)^{\wedge}(x) d x \\
& =-\frac{\pi}{2} \int_{S^{n-1}}\left(|(x, \xi)||x|^{-m-2}\right)^{\wedge}(\theta) D^{m} f^{e}(\theta) d \theta .
\end{aligned}
$$

In case $m \geq n-1, D^{m} f^{e}$ is homogeneous of degree $-1-m \leq-n$, and so we will use (8) or (9) with $p=n-m-1$ to compute its Fourier transform. Thus, for $m-n$ even,

$$
\left(D^{m} f^{e}\right)^{\wedge}(x)=c_{1} \int_{S^{n-1}}|(x, \theta)|^{-n+m+1} D^{m} f^{e}(\theta) d \theta,
$$

and, for $m-n$ odd,

$$
\left(D^{m} f^{e}\right)^{\wedge}(x)=c_{1} \int_{S^{n-1}}\left(C_{n-m-1}-\ln |(x, \theta)|\right)|(x, \theta)|^{-n+m+1} D^{m} f^{e}(\theta) d \theta .
$$

As usual, the indicated constants vary from line to line but always depend only on $m, n$. In order to compute the Fourier transform of $|x|^{-m-2}\left(D^{m} f^{e}\right)^{\wedge}(x)$, we will therefore have to consider two cases according to the parity of $m-n$. 
For $m-n$ even, $|x|^{-m-2}\left(D^{m} f^{e}\right)^{\wedge}(x)$ is homogeneous of degree $-n-1$ and so it follows from (2) and (9) that

$$
\left(|x|^{-m-2}\left(D^{m} f^{e}\right)^{\wedge}(x)\right)^{\wedge}(\xi)=c_{1} \int_{S^{n-1}}|(x, \xi)| \int_{S^{n-1}}|(x, \theta)|^{-n+m+1} D^{m} f^{e}(\theta) d \theta d x
$$

Thus Fubini's theorem and (9) again give

$$
\left(|x|^{-m-2}\left(D^{m} f^{e}\right)^{\wedge}(x)\right)^{\wedge}(\xi)=c_{1} \int_{S^{n-1}}\left(|(x, \xi)||x|^{-m-2}\right)^{\wedge}(\theta) D^{m} f^{e}(\theta) d \theta
$$

The final step is to deal with $m-n \geq-1$ and odd. Here,

$$
\begin{aligned}
& |x|^{-m-2}\left(D^{m} f^{e}\right)^{\wedge}(x) \\
& \begin{array}{l}
=c_{1}|x|^{-n-1} \int_{S^{n-1}}\left(C_{n-m-1}-\ln |(x /|x|, \theta)|\right)|(x /|x|, \theta)|^{-n+m+1} D^{m} f^{e}(\theta) d \theta \\
\quad-c_{1}|x|^{-n-1} \ln |x| \int_{S^{n-1}}|(x /|x|, \theta)|^{-n+m+1} D^{m} f^{e}(\theta) d \theta .
\end{array}
\end{aligned}
$$

The first term is a homogeneous, degree $-n-1$, function and its Fourier transform can be computed by formula (2). The second term has the form $|x|^{-n-1} g(x /|x|) \ln |x|$ for some even function $g$. In order to compute its Fourier transform, we first note that formula (1) holds for all non-integer values of $p<1$. Thus, for small $\alpha$,

$$
\left(|x|^{-n-1+\alpha} g(x /|x|)\right)^{\wedge}(\xi)=\Gamma(-1+\alpha) \sin \frac{\alpha \pi}{2} \int_{S^{n-1}}|(u, \xi)|^{1-\alpha} g(u) d u
$$

The coefficient of the latter integral is an analytic function at $\alpha=0$. Therefore, differentiating both sides with respect to $\alpha$ and letting $\alpha=0$, we get

$$
\begin{aligned}
\left(|x|^{-n-1} \ln |x| g(x /|x|)\right)^{\wedge} & (\xi) \\
=\frac{d}{d \alpha}\left[\Gamma(-1+\alpha) \sin \frac{\alpha \pi}{2}\right]_{\alpha=0} \int_{S^{n-1}}|(u, \xi)| g(u) d u & \\
& +\frac{\pi}{2} \int_{S^{n-1}}|(u, \xi)| \ln |(u, \xi)| g(u) d u \\
& =\int_{S^{n-1}}\left(c_{3}+c_{4} \ln |(u, \xi)|\right)|(u, \xi)| g(u) d u
\end{aligned}
$$

Now, putting everything together, we get

$$
\begin{aligned}
& \left(|x|^{-m-2}\left(D^{m} f^{e}\right)^{\wedge}(x)\right)^{\wedge}(\xi) \\
& =-\frac{\pi}{2} c_{1} \int_{S^{n-1}}|(u, \xi)| \int_{S^{n-1}}\left(C_{n-m-1}-\ln |(u, \theta)|\right)|(u, \theta)|^{-n+m+1} D^{m} f^{e}(\theta) d \theta d u \\
& \quad-c_{1} \int_{S^{n-1}}\left(c_{2}+c_{3} \ln |(u, \xi)|\right)|(u, \xi)| \int_{S^{n-1}}|(u, \theta)|^{-n+m+1} D^{m} f^{e}(\theta) d \theta d x .
\end{aligned}
$$


Fubini's theorem and formula (8) give

$$
\begin{aligned}
\left(|x|^{-m-2}\left(D^{m} f^{e}\right)^{\wedge}(x)\right)^{\wedge}(\xi) & \\
= & -\frac{\pi}{2} c_{1} \int_{S^{n-1}} D^{m} f^{e}(\theta) \int_{S^{n-1}}\left(C_{n-m-1}-\ln |(u, \theta)|\right)|(u, \theta)|^{-n+m+1}|(u, \xi)| d u d \theta \\
& -c_{1} \int_{S^{n-1}} D^{m} f^{e}(\theta) \int_{S^{n-1}}\left(c_{2}+c_{3} \ln |(u, \xi)|\right)|(u, \xi)||(u, \theta)|^{-n+m+1} d u d \theta \\
= & -\frac{\pi}{2} \int_{S^{n-1}}\left(|(x, \xi)||x|^{-m-2}\right)^{\wedge}(\theta) D^{m} f^{e}(\theta) d \theta \\
& -c_{1} \int_{S^{n-1}} D^{m} f^{e}(\theta) \int_{S^{n-1}}\left(c_{2}+c_{3} \ln |(u, \xi)|\right)|(u, \xi)||(u, \theta)|^{-n+m+1} d u d \theta .
\end{aligned}
$$

The second integral is an infinitely differentiable function of $\xi$ in $\mathbb{R}^{n} \backslash o$, since we can write, interchanging $\xi$ and $\theta$,

$$
\begin{aligned}
& \int_{S^{n-1}}\left(c_{2}+\right.\left.c_{3} \ln |(u, \xi)|\right)|(u, \xi)||(u, \theta)|^{-n+m+1} d u \\
&=|\xi| \int_{S^{n-1}}\left(c_{2}+c_{3} \ln |(u, \xi /|\xi|)|\right)|(u, \xi /|\xi|)||(u, \theta)|^{-n+m+1} d u \quad+c_{3}|\xi| \ln |\xi| \int_{S^{n-1}}|(u, \xi /|\xi|)||(u, \theta)|^{-n+m+1} d u \\
&=|\xi| \int_{S^{n-1}}\left(c_{2}+c_{3} \ln |(u, \theta)|\right)|(u, \theta)||(u, \xi /|\xi|)|^{-n+m+1} d u \\
& \quad+c_{3}|\xi| \ln |\xi| \int_{S^{n-1}}|(u, \theta)||(u, \xi /|\xi|)|^{-n+m+1} d u
\end{aligned}
$$

Now, $-n+m+1$ is even and so $|(u, \xi)|^{-n+m+1}$ is a polynomial in $\xi$.

Summarizing, we see that, for the case $m-n \leq-2$ and the case $m-n \geq-1$ and even, we have

$$
\left(|x|^{-m-2}\left(D^{m} f^{e}\right)^{\wedge}(x)\right)^{\wedge}(\xi)=c \int_{S^{n-1}}\left(|(x, \xi)||x|^{-m-2}\right)^{\wedge}(\theta) D^{m} f^{e}(\theta) d \theta
$$

and for the case $m-n \geq-1$ and odd we have

$$
\begin{aligned}
\left(|x|^{-m-2}\left(D^{m} f^{e}\right)^{\wedge}(x)\right)^{\wedge}(\xi) & \\
& =\int_{S^{n-1}}\left[c\left(|(x, \xi)||x|^{-m-2}\right)^{\wedge}(\theta)+F(\theta, \xi)\right] D^{m} f^{e}(\theta) d \theta,
\end{aligned}
$$

where $F$ is an infinitely differentiable function of $\xi$ in $\mathbb{R}^{n} \backslash o$.

It is clear that, for $\theta, \xi \in S^{n-1},\left(|(x, \xi)||x|^{-m-2}\right)^{\wedge}(\theta)$ is a function only of the scalar product $s=(\theta, \xi)$. Our previous arguments show that the dominant term of $\Delta_{\xi}^{m / 2}\left(|(x, \xi)||x|^{-m-2}\right)^{\wedge}(\theta)$ is of the same order as the dominant term of $\left(|(x, \xi)||x|^{-2}\right)^{\wedge}(\theta)$, namely $(1-s)^{-(n-3) / 2}$. Defining $F$ to be identically zero for $m-n \leq-2$ and for $m-n \geq-1$ and even, this completes the proof of (27) for functions $f \in C^{\infty}\left(S^{n-1}\right)$ with $g_{m, n}$ defined by (28). The general result follows by approximation, using the integrability of $g_{m, n}(\xi, \cdot)$ and the fact that $C^{\infty}\left(S^{n-1}\right)$ is dense in $C^{m, \alpha}\left(S^{n-1}\right)$. 
Step 2. It only remains to show that $g_{m, n}$ satisfies a differential equation analogous to (26). In fact, it is clear that the smooth function $F$ in (28) plays no role in the regularity considerations, so it will suffice to show that

$$
h_{m, n}((\theta, \xi))=\Delta_{\xi}^{m / 2}\left(|(x, \xi)||x|^{-m-2}\right)^{\wedge}(\theta)
$$

satisfies the equation

$$
(1-m)(n-m-1) h_{m, n}(s)-(n-1) s h_{m, n}^{\prime}(s)+\left(1-s^{2}\right) h_{m, n}^{\prime \prime}(s)=Q(s),
$$

for $s \in(-1,1)$, where $Q$ is a polynomial. To this end, we will compute

$$
\Delta_{\xi} h_{m, n}((\theta, \xi))=\Delta_{\xi}^{(m+2) / 2}\left(|(x, \xi)||x|^{-m-2}\right)^{\wedge}(\theta) .
$$

By (21) the spherical harmonic expansion of $|(x, \xi)|$ is

$$
-\frac{2}{\pi n \kappa_{n}} \sum_{\substack{k=0 \\ k \text { even }}}^{\infty} N(n, k) \lambda_{k}(n,-1) P_{k}^{n}((x, \xi)) .
$$

We use this and (20), in the case $k>m+1-n$, to see that the spherical harmonic expansion of $\left(|(x, \xi)||x|^{-m-2}\right)^{\wedge}(\theta)$ is

$$
\begin{aligned}
\sum_{\substack{k=0 \\
k \text { even }}}^{m+1-n} a_{k}(m, n) P_{k}^{n}((\theta, \xi)) & \\
& -\frac{2}{\pi n \kappa_{n}} \sum_{\substack{k=m+2-n \\
k \text { even }}}^{\infty} N(n, k) \lambda_{k}(n,-1) \lambda_{k}(n, n-m-1) P_{k}^{n}((\theta, \xi)),
\end{aligned}
$$

where the $a_{k}$ are coefficients whose precise value is not important. Of course, in the case $m \leq n-2$, the first summation is absent.

We now consider the action of $\Delta_{\xi}^{(m+2) / 2}$ on $\left(|(x, \xi)||x|^{-m-2}\right)^{\wedge}(\theta)$, which is a homogeneous degree 1 function in $\xi$. The spherical harmonics, extended homogeneously, are eigenvalues of this operator. To see this, recall that

$$
\Delta\left(H_{k}(\xi /|\xi|)|\xi|^{p}\right)=\Delta_{S} H_{k}(\xi)+p(n+p-2) H_{k}(\xi),
$$

where $\Delta_{S}$ is the spherical Laplacian and the equation is assumed to be restricted to the sphere; see [17, Theorem 1.2.9], for example. Then, since

$$
\Delta_{S} H_{k}(\xi)=-k(k+n-2) H_{k}(\xi)
$$

we have

$$
\Delta\left(H_{k}(\xi /|\xi|)|\xi|^{p}\right)=(p-k)(p+k+n-2) H_{k}(\xi) .
$$

Consequently,

$$
\begin{aligned}
\Delta_{\xi}^{(m+2) / 2}\left(H_{k}(\xi /|\xi|)|\xi|\right) & =\prod_{\substack{l=0 \\
l \text { even }}}^{m}(1-k-l)(1+k+n-2-l) H_{k}(\xi) \\
& =\frac{(-1)^{(m+2) / 2} \lambda_{k}(n, m+1)}{\lambda_{k}(n,-1)} H_{k}(\xi) .
\end{aligned}
$$



sion

It follows that $\Delta_{\xi}^{(m+2) / 2}\left(|(x, \xi)||x|^{-m-2}\right)^{\wedge}(\theta)$ has the spherical harmonic expan-

$$
\begin{aligned}
& \sum_{\substack{k=0 \\
k \text { even }}}^{m+1-n} \frac{(-1)^{(m+2) / 2} \lambda_{k}(n, m+1) a_{k}(m, n)}{\lambda_{k}(n,-1)} P_{k}^{n}((\theta, \xi)) \\
& \quad+\frac{2(-1)^{m / 2}}{\pi n \kappa_{n}} \sum_{\substack{k=m+2-n \\
k \text { even }}}^{\infty} N(n, k) \lambda_{k}(n, m+1) \lambda_{k}(n, n-m-1) P_{k}^{n}((\theta, \xi)) \\
& \quad=\sum_{\substack{k=0 \\
k \text { even }}}^{m+1-n} b_{k}(m, n) P_{k}^{n}((\theta, \xi))+\frac{2(-1)^{m / 2}}{\pi n \kappa_{n}}(2 \pi)^{n} \sum_{\substack{k=m+2-n \\
k \text { even }}}^{\infty} N(n, k) P_{k}^{n}((\theta, \xi)),
\end{aligned}
$$

where we used the fact that $\lambda_{k}(n, m+1) \lambda_{k}(n, n-m-1)=(2 \pi)^{n}$.

The above expression is (up to finitely many terms) an expansion for the even part of the Dirac measure on the sphere. Therefore, for all $\xi \neq \pm \theta$,

$$
\Delta_{\xi} h_{m, n}((\theta, \xi))=\Delta_{\xi}^{(m+2) / 2}\left(|(x, \xi)||x|^{-m-2}\right)^{\wedge}(\theta)=Q((\theta, \xi)),
$$

where $Q$ is a polynomial.

Using the formula (18), we therefore get

$$
\begin{aligned}
\Delta_{\xi}\left(|\xi|^{1-m} h_{m, n}(s)\right)= & \left(1-s^{2}\right) h_{m, n}^{\prime \prime}(s) \\
& -(n-1) s h_{m, n}^{\prime}(s)-(m-1)(n-m-1) h_{m, n}(s)=Q(s),
\end{aligned}
$$

provided $s=(\theta, \xi)$ belongs to the interval $(-1,1)$, where $\xi, \theta \in S^{n-1}$.

It follows immediately from Theorem 6.1 that, for any function $f \in C^{1}\left(S^{n-1}\right) \subset$ $C^{0, \alpha}\left(S^{n-1}\right)$ for all $0<\alpha<1$, we have If $\in C^{2}\left(S^{n-1}\right) \supset C^{2, \beta}\left(S^{n-1}\right)$ for all $0<\beta<1$, where

$$
I f(\xi)=\int_{S^{n-1}} g_{n}((\theta, \xi)) f(\theta) d \theta
$$

Furthermore, the proof of the theorem shows that the above mapping $I: C^{1}\left(S^{n-1}\right)$ $\rightarrow C^{2}\left(S^{n-1}\right)$ is continuous with respect to the usual topologies. This observation, which was kindly pointed out to us by Markus Kiderlen, allows us to obtain a strengthened version of one of his results 23 .

If $f \in C^{1}\left(S^{n-1}\right)$, then $h=I f \in C^{2}\left(S^{n-1}\right)$ is a difference of support functions; see [31, Lemma 1.7.9] for example. Thus $\square h$ is a difference of first surface area measures. If we further assume that $f$ satisfies (11), then $\square h=f$, and so $f$ is a difference of first surface area measures. It follows that the index $2[(n+1) / 4]$ in [23, Proposition 3.2] can be replaced by 1.

In a similar direction, we can obtain a strengthening of a result of Weil [33. He showed that differences of first (and therefore $j$-th) surface area measures are dense, in the weak topology, in the set of signed Borel measures on $S^{n-1}$ with centroid at the origin. This result can now be seen to follow from the observation that functions in $C^{\alpha}\left(S^{n-1}\right)$, for $\alpha>0$, satisfying (11), are differences of first surface area measures.

\section{REFERENCES}

[1] A. D. Aleksandrov, Über die Frage nach der Existenz eines Körpers bei dem die Summe der Hauptkrümmungsradien eine gegebene positive Funktion ist, welche die Bedingungen der Geschlossenheit genügt, Doklady Akad. Nauk 14 (1937), 59-60. 
[2] A. D. Aleksandrov, Zur Theorie der gemischten Volumina von konvexen Körpern, II. Neue Ungleichungen zwischen den gemischten Volumina und ihre Anwendungen (in Russian), Mat. Sbornik N.S. 2 (1937), 1205-1238.

[3] A. D. Aleksandrov, Zur Theorie der gemischten Volumina von konvexen Körpern, III. Die Erweiterung zweier Lehrsätze Minkowskis über die konvexen Polyeder auf beleibige konvexe Flächen (in Russian), Mat. Sbornik N.S. 3 (1938), 27-46.

[4] C. Berg, Corps convexes et potentiels sphériques (French), Mat.-Fys. Medd. Danske Vid. Selsk. 37 (1969) no. 6. MR.0254789 (40:7996)

[5] H. Busemann, Convex surfaces, Interscience Tracts in Pure and Applied Mathematics, no. 6, Interscience Publishers, Inc., New York, 1958. MR0105155 (21:3900)

[6] S.-Y. Cheng, S.-T. Yau, On the regularity of the solution of the $n$-dimensional Minkowski problem, Commun. Pure Appl. Math. 29 (1976), 495-516. MR0423267 (54:11247)

[7] E. B. Christoffel, Über die Bestimmung der Gestalt einer krummen Oberfläche durch lokale Messungen auf derselben, J. für die Reine und Angewandte Math. 64 (1865), 193-209.

[8] W. Fenchel, B. Jessen, Mengenfunktionen und konvexe Körper, Danske Vid. Selskab. Mat.fys. Medd. 16 (1938).

[9] W. J. Firey, The determination of convex bodies from their mean radius of curvature functions, Mathematika 14 (1967), 1-13. MR0217699(36:788)

[10] W. J. Firey, Christoffel's problem for general convex bodies, Mathematika 15 (1968), 7-21. MR0230259 (37:5822)

[11] I. M. Gelfand, G. E. Shilov, Generalized functions, Vol. 1 Properties and Operations, Academic Press, New York and London, 1964. MR0435831 (55:8786a)

[12] D. Gilbarg, N. Trudinger, Elliptic partial differential equations of second order, Springer Verlag, Berlin, 1983. MR737190 (86c:35035)

[13] H. Gluck, Manifolds with preassigned curvature - a survey, Bull. Amer. Math.Soc. 81 (1975), 313-329. MR0367861 (51:4103)

[14] P. Goodey, W. Weil, Centrally symmetric convex bodies and the spherical Radon transforms, J. Diff. Geom. 35 (1992), 675-688. MR1163454 (93g:44005)

[15] P. Goodey, V. Yaskin, M. Yaskina, Fourier transforms and the Funck-Hecke theorem in convex geometry, J. London Math. Soc. (2) 80 (2009), 388-404. MR2545259

[16] I. S. Gradshteyn, I. M. Ryzhik, Tables of integrals, series and products, 7th edition, Academic Press, 2007. MR2360010 (2008g:00005)

[17] H. Groemer, Geometric applications of Fourier series and spherical harmonics, Cambridge Univ. Press, New York, 1996. MR1412143 (97j:52001)

[18] B. Guan, P. Guan, Convex hypersurfaces of prescribed curvatures, Ann. of Math. (2) 156 (2002), 655-673. MR1933079 (2003i:53046)

[19] P. Guan, C. Lin, X. Ma, The Christoffel-Minkowski problem II. Weingarten curvature equations, Chinese Ann. Math. Ser. B 27 (2006), 595-614. MR2273800(2007k:35145)

[20] P. Guan, X. Ma, The Christoffel-Minkowski problem I. Convexity of solutions if a Hessian equation, Invent. Math. 151 (2003), 553-577. MR1961338(2004a:35071)

[21] P. Guan, X. Ma, Convex solutions of fully nonlinear elliptic equations in classical differential geometry. Geometric evolution equations, 115-127, Contemp. Math. 367 (2005). MR 2115755 (2006a:35085)

[22] P. Guan, X. Ma, F. Zhou, The Christoffel-Minkowski problem III. Existence and convexity of admissible solutions, Comm. Pure Appl. Math. 59 (2006), 1352-1376. MR2237290 (2007m:35064)

[23] M. Kiderlen, Stability results for convex bodies in geometric tomography, Indiana Univ. Math. Jour. 57, No. 5 (2008), 1999-2038. MR2463960(2010c:52003)

[24] A. Koldobsky, Fourier Analysis in Convex Geometry, Mathematical Surveys and Monographs, American Mathematical Society, Providence RI, 2005. MR2132704 (2006a:42007)

[25] E. Milman, Generalized intersection bodies, J. Funct. Anal. 240 (2006), 530-567. MR2261694 (2007h:52007)

[26] H. Minkowski, Allgemeine Lehrsätze über die konvexen Polyeder, Nachr. Ges. Wiss. Göttingen (1897), 198-219. Gesammelte Abhandlungen, vol. II, Teubner, Leipzig, 1911, 103121.

[27] H. Minkowski, Volumen und Oberfläche, Math. Ann. 57 (1903), 447-495. Gesammelte Abhandlungen, vol. II, Teubner, Leipzig, 1911, 230-276. MR.1511220 
[28] A. V. Pogorelov, The Minkowski Multidimensional Problem, Winston and Sons, Washington DC, 1978. MR 0478079 (57:17572)

[29] W. Rudin, Functional analysis, McGraw-Hill, New York, 1973. MR0365062 (51:1315)

[30] R. Schneider, Das Christoffel-Problem für Polytope, Geom. Dedicata 6 (1977), 81-85. MR 0470865 (57:10609)

[31] R. Schneider, Convex Bodies: the Brunn-Minkowski Theory, Cambridge University Press, Cambridge, 1993. MR 1216521 (94d:52007)

[32] W. Sheng, N. Trudinger, X. Wang, Convex hypersurfaces of prescribed Weingarten curvature, Comm. in Analysis and Geometry, 12 (2004), 213-232. MR2074877(2005f:53109)

[33] W. Weil, On surface area measures of convex bodies, Geom. Dedicata 9 (1980), 299-306. MR:585937 (81m:52014)

Department of Mathematics, University of Oklahoma, Norman, Oklahoma 73019

E-mail address: pgoodey@math.ou.edu

Department of Mathematical and Statistical Sciences, University of Alberta, Edmonton, Alberta, Canada T6G 2G1

E-mail address: vladyaskin@math.ualberta.ca

Department of Mathematical and Statistical Sciences, University of Alberta, Edmonton, Alberta, Canada T6G 2G1

E-mail address: myaskina@math.ualberta.ca 